\title{
Two asymptotic problems for a singular nonlinear Schrödinger system
}

\author{
Nader Masmoudi* Kenji Nakanishi ${ }^{\dagger}$
}

June 18, 2008

\section{Abstract}

In this paper, we continue our investigation of the relation between various systems that can be derived from the Klein-Gordon-Zakharov system in the high-frequency and subsonic limits. In this paper we start from the singular nonlinear Schrödinger system which was derived in [15] and derive the classical nonlinear Schrödinger system in two different limit cases. These two nonlinear Schrödinger systems have different coefficients in the nonlinearity.

\section{Introduction}

In [15], we investigated a resonance phenomenon in the high-frequency and subsonic limits of the Klein-Gordon-Zakharov system (KGZ)

$$
\begin{cases}c^{-2} \ddot{E}-\Delta E+c^{2} E=-n E, & E: \mathbb{R}^{1+3} \rightarrow \mathbb{R}^{3}, \\ \alpha^{-2} \ddot{n}-\Delta n=\Delta|E|^{2}, & n: \mathbb{R}^{1+3} \rightarrow \mathbb{R},\end{cases}
$$

where $E$ and $n$ describe the Langmuir oscillations and the ion sound waves in plasma, $c^{2}$ is the plasma frequency and $\alpha$ is the ion sound speed. Under the resonance condition $\alpha \gamma=2 c^{2}$ with arbitrary fixed $\gamma$, we derived the following limit system :

$$
2 i \dot{\mathbb{E}}-\Delta \mathbb{E}=|\mathbb{E}|^{2} \mathbb{E}+\frac{1}{2} \mathcal{A}_{\gamma}\left(\mathbb{E} \cdot \mathbb{E}^{\perp}\right) \overline{\mathbb{E}^{\perp}}
$$

where $\mathbb{E}=\left(\mathbb{E}_{1}, \mathbb{E}_{2}\right): \mathbb{R}^{1+3} \rightarrow \mathbb{C}^{3} \times \mathbb{C}^{3}$ comes from the two modes of oscillations in $E$ of $\mathrm{KGZ}, \mathbb{E}^{\perp}=\left(\mathbb{E}_{2}, \mathbb{E}_{1}\right)$, and $\mathcal{A}_{\gamma}$ denotes the following singular operator

$$
\mathcal{A}_{\gamma}=P V\left(\frac{|\nabla|^{2}}{|\nabla|^{2}-\gamma^{2}}\right)-\frac{i \gamma \pi}{2} \delta_{|\nabla|=\gamma}=\frac{1}{2}\left\{\frac{|\nabla|}{|\nabla|+\gamma}+\frac{|\nabla|}{|\nabla|-\gamma+i 0}\right\},
$$

${ }^{*}$ Courant Institute, New York University, 251 Mercer street, New York, NY 10012, USA. Partially supported by an NSF grant Grant No DMS-0703145.

$\dagger$ Department of Mathematics, Kyoto University, Kyoto 606-8502, Japan 
which comes from a careful analysis of the resonances in KGZ.

In this paper, we will take (1.2) as our starting point and study the two limits $\gamma$ going to 0 and $\gamma$ going to $\infty$. The dimension six of the range of $\mathbb{E}$ is completely irrelevant throughout this paper. Also the transform $\mathbb{E} \mapsto \mathbb{E}^{\perp}$ can be replaced with any complex linear map.

In the limit $\gamma$ going to $\infty$, we prove the convergence towards the following coupled nonlinear Schrödinger system (NLS)

$$
2 i \dot{\mathbb{E}}-\Delta \mathbb{E}=|\mathbb{E}|^{2} \mathbb{E}
$$

This is exactly the system derived in [13] and [14] as the limit of KGZ for $(c, \alpha) \rightarrow \infty$ in the case $\alpha<c$. In the limit $\gamma$ going to 0 , we prove the convergence towards the following coupled nonlinear Schrödinger system

$$
\left\{\begin{array}{l}
2 i \dot{\mathbb{E}}_{1}-\Delta \mathbb{E}_{1}=\left(\left|\mathbb{E}_{1}\right|^{2}+2\left|\mathbb{E}_{2}\right|^{2}\right) \mathbb{E}_{1} \\
2 i \dot{\mathbb{E}}_{2}-\Delta \mathbb{E}_{2}=\left(\left|\mathbb{E}_{2}\right|^{2}+2\left|\mathbb{E}_{1}\right|^{2}\right) \mathbb{E}_{2}
\end{array}\right.
$$

which is the system derived in [11] as the non-relativistic limit of the nonlinear Klein-Gordon equation:

$$
c^{-2} \ddot{E}-\Delta E+c^{2} E=|E|^{2} E,
$$

which can be derived from KGZ if we send $\alpha$ to $\infty$ while keeping $c$ fixed [3].

Our goals in this paper are to rigorously affirm that nonlinear resonances can lead to different NLS's depending on the way (order) of taking the limits, and to propose the equation (1.2) as a simple model connecting such different limits by one parameter of the resonant frequency.

\section{Preliminaries}

Before stating our main results, we need to introduce several notations. First we define

$$
\begin{aligned}
& \langle a\rangle:=\left(1+|a|^{2}\right)^{1 / 2}, \quad\langle a, b\rangle=\Re(a \cdot \bar{b}), \\
& \langle f \mid g\rangle_{x}:=\int_{\mathbb{R}^{3}}\langle f(x), g(x)\rangle d x, \quad\langle u \mid v\rangle_{t, x}:=\int_{\mathbb{R}^{1+3}}\langle f(t, x), g(t, x)\rangle d t d x,
\end{aligned}
$$

where $a, b, f, g, u$ and $v$ may be scalar or vector valued.

\subsection{Fourier multipliers and frequency decomposition}

We define the space and the space-time Fourier transform by

$$
\mathcal{F}_{3} \varphi=\widetilde{\varphi}(\xi)=\int_{\mathbb{R}^{3}} \varphi(x) e^{-i x \xi} d x, \quad \mathcal{F}_{4} u=\hat{u}(\tau, \xi)=\int_{\mathbb{R}^{1+3}} u(t, x) e^{-i t \tau-i x \xi} d t d x .
$$


For any function $\varphi$, we define the Fourier multiplier $\varphi(\nabla):=\mathcal{F}_{3}^{-1} \varphi(\xi) \mathcal{F}_{3}$.

Next we introduce smooth cut-off in the frequency. Let $\chi \in C_{0}^{\infty}\left(\mathbb{R}^{n}\right)$ satisfies $0 \leq \chi \leq 1, \chi(\xi)=1$ for $|\xi| \leq 1$ and $\chi(\xi)=0$ for $|\xi| \geq 2$. For any $a>0$ and any function $\varphi$, we denote

$$
f_{\leq a}:=\chi(|\nabla / a|) f, \quad f_{>a}:=f-f_{\leq a}, \quad f_{a<\cdot \leq b}:=f_{\leq b}-f_{\leq a} .
$$

For the singular frequency $\gamma$, we need more precise cut-offs.

Definition 2.1.

$$
f_{\check{\gamma}}=\check{\phi}(|\nabla / \gamma|) f, \quad f_{\hat{\gamma}}=\hat{\phi}(|\nabla / \gamma|) f, \quad f_{\check{X}}=f-f_{\check{\gamma}}, \quad f_{\hat{X}}=f-f_{\hat{\gamma}},
$$

where $\check{\phi}, \hat{\phi} \in C_{0}^{\infty}(\mathbb{R})$ should satisfy

(S1) $\check{\phi}(r)=1$ around $r=1$.

(S2) $\hat{\phi}=1$ on a neighborhood of $\operatorname{supp} \check{\phi}$.

(S3) If $\hat{\phi}\left(r_{1}\right)=\hat{\phi}\left(r_{2}\right)=1$ then $2 r_{2}^{2} \geq r_{2}^{2}$.

To fix the estimates, we choose them such that $\check{\phi}(r)=1$ for $8 / 9 \leq r \leq 10 / 9$, $\operatorname{supp} \check{\phi} \subset(7 / 8,9 / 8), \hat{\phi}(r)=1$ for $6 / 7 \leq r \leq 8 / 7$, and $\operatorname{supp} \hat{\phi} \subset(5 / 6,7 / 6)$.

Also we denote for sufficiently big number $N \gg 1$ (depending on the choice of the above cut-off functions),

$$
f_{\ll a}:=f_{\leq a / N}, \quad f_{\gtrsim a}:=f-f_{\ll a} .
$$

For example, we can choose $N=999$ for the above particular choice of $\check{\phi}$ and $\hat{\phi}$.

\section{$2.2 \quad$ Some spaces}

When dealing with the limit $\gamma$ going to infinity we will work in a space with different Sobolev regularities depending on the frequency. We denote the inhomogeneous Besov space by $B_{p}^{s}=B_{p, 2}^{s}$. For any $s^{\prime} \geq s \geq 0,0<\varepsilon<1$ and $\gamma \gg 1$, we define the space $G_{p}^{\varepsilon, r, s^{\prime}}$ by the norm

$$
\begin{aligned}
\|\varphi\|_{G_{p}^{\varepsilon, s, s^{\prime}}} & :=\left\|\varphi_{\leq \gamma^{\varepsilon}}\right\|_{B_{p}^{s}}+\gamma^{s^{\prime}-r}\left\|\varphi_{\gamma^{\varepsilon}<\leq \gamma}\right\|_{B_{p}^{r}}+\gamma^{s^{\prime}-s}\left\|\varphi_{>\gamma}\right\|_{B_{p}^{s}} \\
& \sim\|\varphi\|_{B_{p}^{s}}+\gamma^{s^{\prime}-r}\left\|\varphi_{\leq \gamma}\right\|_{B_{p}^{r}}+\gamma^{s^{\prime}-s}\left\|\varphi_{>\gamma}\right\|_{B_{p}^{s}},
\end{aligned}
$$

where $r:=\left(s^{\prime}-s \varepsilon\right) /(1-\varepsilon)$ is chosen so that the second norm is larger for $|\xi|>\gamma^{\varepsilon}$ and smaller for $|\xi|<\gamma^{\varepsilon}$ than the first one. On the other hand, the third norm is 
smaller for $|\xi|>\gamma$ and bigger for $|\xi|<\gamma$ than the second one. In particular, we have

$$
B_{p}^{s}=G_{p}^{\varepsilon, s, s} \subset G_{p}^{\varepsilon, s, s^{\prime}}
$$

We will omit $\varepsilon$ and $p=2$, i.e.

$$
G_{p}^{s, s^{\prime}}=G_{p}^{\varepsilon, s, s^{\prime}}, \quad G^{s, s^{\prime}}=G_{2}^{s, s^{\prime}}
$$

We also define the Fourier restriction space $X^{s, b}$ on $\mathbb{R}^{1+3}$ by the norm

$$
\|f\|_{X^{s, b}}=\left\|e^{i t \Delta / 2} f\right\|_{H_{t}^{b} H_{x}^{s}}
$$

and its $\gamma$-dependent version $Y^{s, s^{\prime}, b}$ by

$$
\|f\|_{Y^{s, s^{\prime}, b}}=\left\|e^{i t \Delta / 2} f\right\|_{H_{t}^{b} G_{x}^{s, s^{\prime}}}
$$

Those norms can be defined through the space-time Fourier transform, such as

$$
\|u\|_{X^{s, b}}=\left\|\langle\xi\rangle^{s}\left\langle 2 \tau-|\xi|^{2}\right\rangle^{b} \mathcal{F}_{4} u\right\|_{L^{2} L^{2}}
$$

We also define the space $\mathcal{X}$ by the norm

$$
\|u\|_{\mathcal{X}}=\left\|u_{\hat{X}}\right\|_{Y^{s-1, s^{\prime}-1,1}}+\left\|u_{\hat{\gamma}}\right\|_{X^{s^{\prime}-3 / 2,1}} .
$$

In order to use those norms for the local solutions in a uniform fashion, we extend the integral equation to the whole line. For $0<T<1$, we define the extension operator $\rho_{T}$ from $[0, T]$ to $\mathbb{R}$ by

$$
\rho_{T} f= \begin{cases}f(t) & (0 \leq t \leq T), \\ f(2 T-t) & (T<t \leq 2 T), \\ f(0) & \text { (otherwise) }\end{cases}
$$

and the associated integral operator $\mathcal{I}_{T}$ by

$$
\mathcal{I}_{T} f=\rho_{T} \int_{0}^{t} f(s) d s .
$$

Then we can formulate the extended integral equation for (1.2):

$$
\mathbb{E}=\chi(t) e^{-i t \Delta / 2} \mathbb{E}(0)+\frac{1}{2 i} e^{-i t \Delta / 2} \mathcal{I}_{T} e^{i t \Delta / 2}\left[|\mathbb{E}|^{2} \mathbb{E}+\frac{1}{2} \mathcal{A}_{\gamma}\left(\mathbb{E} \cdot \mathbb{E}^{\perp}\right) \overline{\mathbb{E}^{\perp}}\right]
$$


where $\chi \in C_{0}^{\infty}(\mathbb{R})$ is a fixed cut-off satisfying $\chi(t)=1$ for $|t| \leq 1$. Clearly, any global solution $\mathbb{E}$ of this equation satisfies (1.2) on $[0, T]$. Conversely, given any local solution $\mathbb{E}$ on $[0, T]$, we obtain the unique global solution of the above which is equal to $\mathbb{E}$ on $[0, T]$, just by plugging the local solution into the right hand side.

In the following, our solution $\mathbb{E}$ will be always a global solution of (2.15). For brevity, we will also denote

$$
S_{T}=\frac{1}{2 i} e^{-i t \Delta / 2} \mathcal{I}_{T} e^{i t \Delta / 2}
$$

\section{Main results}

In this section, we state the main results of this paper. We start by the limit $\gamma$ going to infinity and state two results. The first one is set in the Sobolev space $H^{s}$ for $s \geq 3 / 2$.

Theorem 3.1. Let $s \geq 3 / 2$ and $\mathbb{E}^{\gamma}$ be the solution of (1.2), on a maximal time interval $\left[0, T^{\gamma}\right)$, with the initial data $\mathbb{E}^{\gamma}(0)$ such that $\mathbb{E}^{\gamma}(0)$ goes to $\mathbb{E}^{\infty}(0)$ in $H^{s}$ when $\gamma$ goes to infinity. Let $\mathbb{E}^{\infty}$ be the solution of the $N L S$

$$
2 i \dot{\mathbb{E}}-\Delta \mathbb{E}=|\mathbb{E}|^{2} \mathbb{E}
$$

on the maximal time interval $\left[0, T^{\infty}\right)$. Then, we have in the limit

$$
\begin{aligned}
& \liminf T^{\gamma} \geq T^{\infty}, \\
& \mathbb{E}^{\gamma} \rightarrow \mathbb{E}^{\infty} \text { in } C\left([0, T] ; H^{s}\right),
\end{aligned}
$$

for all $0<T<T^{\infty}$.

The second result improves the regularity for the frequencies different from $\gamma$. It is set in the space $G^{s, s^{\prime}}$ for $s \geq 1 / 2$ and $s^{\prime} \geq 3 / 2$. The first theorem is contained as the special case where $s=s^{\prime} \geq 3 / 2$.

Theorem 3.2. Let $s \geq 1 / 2, s^{\prime} \geq \max (3 / 2, s)$ and $0<\varepsilon<1$. Let $\mathbb{E}^{\gamma}$ be the solution of (1.2), on a maximal time interval $\left[0, T^{\gamma}\right)$, with the initial data $\mathbb{E}^{\gamma}(0)$ bounded in $G^{\varepsilon, s, s^{\prime}}$ and converging to $\mathbb{E}^{\infty}(0)$ in $H^{s}$ as $\gamma$ goes to infinity. Let $\mathbb{E}^{\infty}$ be the solution of the nonlinear Schrödinger system (3.1) on the maximal time interval $\left[0, T^{\infty}\right)$. Then, we have in the limit $\lim \inf T^{\gamma} \geq T^{\infty}$, and for any $0<T<T^{\infty}$,

$$
\mathbb{E}^{\gamma} \rightarrow \mathbb{E}^{\infty} \text { in } C\left([0, T] ; H^{s}\right)
$$

and moreover they are bounded in $C\left([0, T] ; G^{\varepsilon, s, s^{\prime}}\right)$ (with bounds depending on $T$ ). 
The unconditional uniqueness for the cubic NLS in $H^{1 / 2}\left(\mathbb{R}^{3}\right)$ is an open question. Hence the maximal existence time in this case should be defined in the restricted solution class with finite Strichartz norms. Specifically, we have for all $T \in\left(0, T^{\infty}\right)$,

$$
\mathbb{E}^{\infty} \in C\left([0, T] ; H^{s}\right) \cap L^{4} L^{6}(0, T),
$$

and if $\mathbb{E}^{\infty} \in L^{4} L^{6}\left(0, T^{\infty}\right)$ then $T^{\infty}=\infty$. The $L^{4} L^{6}$ norm is controlled by the $H^{s}$ norm in the case $s>1 / 2$. Moreover the bound on $\left\|\mathbb{E}^{\gamma}\right\|_{L^{\infty}\left(0, T ; G^{\varepsilon, s, s^{\prime}}\right)}$ can be given in terms of the initial bound and $\left\|\mathbb{E}^{\infty}\right\|_{L^{\infty}\left(0, T ; H^{s}\right) \cap L^{4} L^{6}(0, T)}$.

It is in general easy to consider stronger convergences. In particular, if we assume that initially the second and the third components in the $G^{\varepsilon, s, s^{\prime}}$ norm (2.6) go to 0 , then it persists for later time up to the maximal existence of the limit solution. That can be seen in the later estimates, just by dropping the first component in the second line of (2.6) whenever we apply that norm to functions with the highest frequency.

The next result concerns the convergence when $\gamma$ goes to 0 .

Theorem 3.3. Let $s \geq 1 / 2$ and $\mathbb{E}^{\gamma}$ be the solution of (1.2), on a maximal time interval $\left[0, T^{\gamma}\right)$, with the initial data $\mathbb{E}^{\gamma}(0)$ such that $\mathbb{E}^{\gamma}(0)$ goes to $\mathbb{E}^{\infty}(0)$ in $H^{s}$ when $\gamma$ goes to 0 . Let $\mathbb{E}^{\infty}$ be the solution of the $N L S$

$$
2 i \dot{\mathbb{E}}-\Delta \mathbb{E}=|\mathbb{E}|^{2} \mathbb{E}+\frac{1}{2}\left(\mathbb{E} \cdot \mathbb{E}^{\perp}\right) \overline{\mathbb{E}^{\perp}}
$$

on the maximal time interval $\left[0, T^{\infty}\right)$. Then, we have in the limit

$$
\begin{aligned}
& \liminf T^{\gamma} \geq T^{\infty}, \\
& \mathbb{E}^{\gamma} \rightarrow \mathbb{E}^{\infty} \text { in } C\left([0, T] ; H^{s}\right),
\end{aligned}
$$

for all $0<T<T^{\infty}$.

The coefficients in the second limit NLS was written more explicitly in (1.5).

The rest of the paper is organized as follows. The next 4 sections deal with the limit $\gamma$ going to infinity and more precisely with the proof of Theorem 3.2.

We point out that the critical case, namely $s=1 / 2$ and $s^{\prime}=3 / 2$ requires more work. In subsection 4.1, we estimate the singular term in $L^{4}$ in space. Then, in subsection 4.2 , we prove the Strichartz estimate for the nonresonant part. In section 5 , we prove the estimate in the $\mathcal{X}$ space and in section 6 , we give the energy estimate at the resonant frequency, thereby concluding uniform boundedness of $\mathbb{E}^{\gamma}$ in subsection 6.1. Section 7 is devoted to the convergence proof.

Finally, Section 8 is devoted to the limit $\gamma \rightarrow 0$ and the proof of Theorem 3.3.

In the appendix, we give two proofs of the $L^{4}$ bound on the operator $\mathcal{A}_{\gamma}$. 


\section{Strichartz estimate at the nonresonant frequency}

We start here the proof of Theorem 3.2. In this section and the next two ones, we will give uniform estimates which yield the existence of a solution to (1.2) on some small time interval $(0, T)$ which is independent of $\gamma$. The goal of this section and sections 5 and 6 is to estimate those norms

$$
\left\|\mathbb{E}_{\hat{X}}\right\|_{L^{2} G_{6}^{s, s^{\prime}}(0, T)}, \quad\|\mathbb{E}\|_{\mathcal{X}(0, T)}, \quad\|\mathbb{E}\|_{L^{\infty}\left(0, T ; G^{s, s^{\prime}}\right)}
$$

uniformly in $\gamma$ on a fixed short time interval $[0, T]$.

The most difficult case is the critical case, namely $s=1 / 2$ and $s^{\prime}=3 / 2$. In particular there is a difficulty related to the lack of the $L^{\infty}$ estimate for the low frequency part due to the failure of the Sobolev embedding of $B_{6}^{1 / 2}$ into $L^{\infty}$. We start here by estimating $\left\|\mathbb{E}_{\hat{X}}\right\|_{L^{2} G_{6}^{s, s^{\prime}}(0, T)}+\left\|\mathbb{E}_{\hat{X}}\right\|_{L^{\infty}\left(0, T ; G^{s, s^{\prime}}\right)}$.

\subsection{Linear and quadratic estimates}

Proposition 4.1. If $E \in L^{\infty}\left(0, T ; G^{s, s^{\prime}}\right)$ and $E_{\hat{X}} \in L^{2} G_{6}^{s, s^{\prime}}(0, T)$, then we have

$$
\begin{aligned}
& \|E\|_{L^{4} L^{6,2}} \lesssim T^{1 / 4} \gamma^{-1 / 2}\left\|E_{\hat{\gamma}}\right\|_{L^{\infty} H^{s^{\prime}}}+\left\|E_{\hat{X}}\right\|_{L^{\infty} H^{1 / 2}}^{1 / 2}\left\|E_{\hat{X}}\right\|_{L^{2} B_{6}^{1 / 2}}^{1 / 2}, \\
& \|E\|_{L^{8} L^{4}} \lesssim\|E\|_{L^{\infty} H^{1 / 2}}^{1 / 2}\|E\|_{L^{4} L^{6,2}}^{1 / 2}, \\
& \left\|\mathcal{A}_{\gamma}\left(E^{2}\right)_{\check{\gamma}}\right\|_{L^{8} B_{4}^{s^{\prime}-3 / 2}} \lesssim\|E\|_{L^{8} L^{4}}\left\|E_{\gtrsim_{\gamma}}\right\|_{L^{\infty}\left(\gamma^{\left.s-s^{\prime} H^{s}\right)}\right.},
\end{aligned}
$$

where $L^{p, q}$ denotes the Lorentz space.

Trivially we may replace $L^{\infty} H^{1 / 2}$ with $L^{\infty} G^{s, s^{\prime}}$ and $L^{2} B_{6}^{1 / 2}$ by $L^{2} G_{6}^{s, s^{\prime}}$. Also remember that the last norm is the component for $|\xi| \geq \gamma$ in the norm $G_{2}^{s, s^{\prime}}$.

Proof. For the first inequality, we just use the Sobolev embedding for $E_{\hat{\gamma}}$. For $E_{\hat{X}}$, we use the complex interpolation and then the Sobolev embedding (into the Lorentz space for the first inequality). The second inequality follows from Hölder after the embeddings $H^{1 / 2} \subset L^{3}$ and $L^{6,2} \subset L^{6}$.

For the estimate of $\mathcal{A}_{\gamma}\left(E^{2}\right)_{\check{\gamma}}$, we use the fact that, when restricted to frequencies of order $\gamma, \mathcal{A}_{\gamma}$ is bounded from $L^{4 / 3}$ to $L^{4}$ with a norm bounded by $\gamma^{3 / 2}$ (cf. Appendix). Notice that $\left(E^{2}\right)_{\check{\gamma}}$ is bounded in $\gamma^{-s^{\prime}} L^{8} L^{4 / 3}$. Indeed, expanding $E^{2}$ by $E=E_{\ll \gamma}+E_{\gtrsim \gamma}$, we have $\left(E_{\ll \gamma}\right)_{\check{\gamma}}^{2}=0$, and the other terms are bounded by putting $E_{\gtrsim \gamma}$ in $L^{\infty}\left(\gamma^{s-s^{\prime}} H^{s}\right)$ and the other function in $L^{8} L^{4}$. 


\subsection{Cubic estimates}

Proposition 4.2. If $E \in L^{\infty}\left(0, T ; G^{s, s^{\prime}}\right)$ and $E_{\hat{X}} \in L^{2} G_{6}^{s, s^{\prime}}(0, T)$, then we have

$$
\begin{aligned}
& \left\|\left(E^{2}+\mathcal{A}_{\gamma}\left(E^{2}\right)_{\check{X}}\right) \bar{E}\right\|_{L^{2} G_{6 / 5}^{s, s^{\prime}}} \lesssim\|E\|_{L^{\infty} G^{s, s^{\prime}}}\|E\|_{L^{4} L^{6}}^{2}, \\
& \left\|\left[\mathcal{A}_{\gamma}\left(E^{2}\right)_{\check{\gamma}} \bar{E}\right]_{\hat{X}}\right\|_{L^{8} G_{4 / 3}^{s, s^{\prime}}} \lesssim\left\|E_{\gtrsim \gamma}\right\|_{L^{\infty}\left(\gamma^{\left.s-s^{\prime} H^{s}\right)}\right.}^{2}\|E\|_{L^{8} L^{4}}
\end{aligned}
$$

Proof. The terms $E^{2} \bar{E}$ are estimated by the standard Littlewood-Paley decomposition of the three functions, putting the highest frequency part in $G^{s, s^{\prime}}$ and the other two in $L^{6}$.

Remark that for the high-high interactions, namely when the product has lower frequency than the highest one, we get even better decay for $G_{p}^{s, s^{\prime}}$ compared with the standard $B_{p}^{s}$. For example, if the highest frequency $k$ is put in the third component in the norm (2.6) and the product is in the second one, then the gain is

$$
(j / \gamma)^{r}(k / \gamma)^{-s}=(j / k)^{-s}(j / \gamma)^{r-s} \leq(j / k)^{-s}
$$

where on the right we put the gain in the case of $B_{p}^{s}$ for comparison.

The term with $\mathcal{A}_{\gamma}\left(E^{2}\right)_{\check{X}}$ is treated in the same way, because the operator $\mathcal{A}_{\gamma}$ restricted away from the singular frequency $\gamma$ is uniformly bounded in any Besov space. Integrating in time, we get the first two inequalities in (4.4).

Now we treat the term with $\mathcal{A}_{\gamma}\left(E^{2}\right)_{\check{\gamma}}$. The point here is that

$$
\left[\mathcal{A}_{\gamma}\left(E^{2}\right)_{\check{\gamma}} \bar{E}_{\ll \gamma}\right]_{\hat{X}}=0
$$

because of their Fourier support ((S2) in Definition 2.1). For the rest, we use (4.3) for the singular part, and put $\bar{E}_{\gtrsim \gamma}$ in $L^{\infty}\left(\gamma^{s-s^{\prime}} H^{s}\right)$, which dominates $G^{s, s^{\prime}}$ in this frequency band.

Finally, combining the above estimates with the Strichartz estimate

$$
\left\|S_{T} f\right\|_{L^{2} B_{6,2}^{s} \cap L^{\infty} H^{s}} \lesssim\|f\|_{L^{2} B_{6 / 5,2}^{s}+L^{8 / 5} B_{4 / 3,2}^{s}}
$$

in different regularities, we deduce the following proposition. The operator $S_{T}$ was defined in (2.16).

Proposition 4.3. If $E \in L^{\infty}\left(0, T ; G^{s, s^{\prime}}\right)$ and $E_{\hat{X}} \in L^{2} G_{6}^{s, s^{\prime}}(0, T)$, then

$$
\begin{aligned}
& \left\|S_{T}\left[\left(E^{2}+\mathcal{A}_{\gamma} E^{2}\right) \bar{E}\right]_{\hat{X}}\right\|_{L^{2} G_{6}^{s, s^{\prime}} \cap L^{\infty} G^{s, s^{\prime}}} \\
& \lesssim\left(T^{1 / 2} \gamma^{-1}+T^{5 / 8} \gamma^{-3 / 4}\right)\|E\|_{L^{\infty} G^{s, s^{\prime}}}^{3}+\|E\|_{L^{\infty} G^{s, s^{\prime}}}\|E\|_{L^{4} L^{6}}^{2} \\
& \quad+T^{1 / 2}\|E\|_{L^{\infty} G^{s, s^{\prime}}}^{5 / 2}\|E\|_{L^{4} L^{6}}^{1 / 2} .
\end{aligned}
$$

For the last term, we applied (4.2) to the $L^{8} L^{4}$ norm. 


\section{The $\mathcal{X}$ estimate}

In this section, we derive the $X^{s, b}$ type estimate. Recall the space $\mathcal{X}$ defined in (2.12).

Proposition 5.1. If $E \in L^{\infty}\left(0, T ; G^{s, s^{\prime}}\right)$ and $E_{\hat{X}} \in S t r^{s, s^{\prime}}(0, T)$, then

$$
\begin{aligned}
& \left\|S_{T}\left[\left(E^{2}+\mathcal{A}_{\gamma} E^{2}\right) \bar{E}\right]\right\|_{\mathcal{X}} \\
& \lesssim T^{1 / 2} \gamma^{-1}\|E\|_{L^{\infty} G^{s, s^{\prime}}}^{3}+\|E\|_{L^{\infty} G^{s, s^{\prime}}}\|E\|_{L^{4} L^{6}}^{2} \\
& \quad+T^{1 / 4}\|E\|_{L^{\infty} G^{s, s^{\prime}}}^{2}\|E\|_{L^{4} L^{6}}+\gamma^{-1 / 4} T^{3 / 8}\|E\|_{L^{\infty} G^{s, s^{\prime}}}^{5 / 2}\|E\|_{L^{4} L^{6}}^{1 / 2} .
\end{aligned}
$$

Proof. The left hand side is bounded by

$$
\left\|\left[\left(E^{2}+\mathcal{A}_{\gamma} E^{2}\right) \bar{E}\right]_{\hat{X}}\right\|_{L^{2} G^{s-1, s^{\prime}-1}}+\left\|\left[\left(E^{2}+\mathcal{A}_{\gamma} E^{2}\right) \bar{E}\right]_{\hat{\gamma}}\right\|_{L^{2} H^{s^{\prime}-3 / 2}} .
$$

Notice that the first norm is stronger than the second at frequency $\gamma$.

First in the cubic term $\left(E^{2}+\mathcal{A}_{\gamma}\left(E^{2}\right)_{\check{X}}\right) \bar{E}$, we put one of the $E$ 's having the highest frequency into $G^{s, s^{\prime}}$ and the two others in $L^{6}$. Hence the product satisfies

$$
\left\|\left(E^{2}+\mathcal{A}_{\gamma}\left(E^{2}\right)_{\check{X}}\right) \bar{E}\right\|_{L^{2} G^{s-1, s^{\prime}-1}} \lesssim\|E\|_{L^{\infty} G^{s, s^{\prime}}}\|E\|_{L^{4} L^{6}}^{2},
$$

where the regularity loss is due to the Sobolev $L^{6 / 5} \subset H^{-1}$.

Next we estimate $\mathcal{A}_{\gamma}\left(E^{2}\right)_{\check{\gamma}} \bar{E}_{\ll \gamma}$, which does not contribute to the $\hat{X}$ frequency thanks to (4.6). Hence we can estimate the product by using (4.3),

$$
\left\|\mathcal{A}_{\gamma}\left(E^{2}\right)_{\grave{\gamma}} \bar{E}_{\ll \gamma}\right\|_{L^{2} H^{s^{\prime}-3 / 2}} \lesssim T^{1 / 4}\left\|E_{\gtrsim \gamma}\right\|_{L^{\infty}\left(\gamma^{\left.s-s^{\prime} H^{s}\right)}\right.}\|E\|_{L^{8} L^{4}}^{2} .
$$

For the remaining term with $\bar{E}_{\gtrsim \gamma}$, we put it in $L^{\infty}\left(\gamma^{s-s^{\prime}} H^{s}\right)$ and use (4.3). Hence the product is in $L^{8} G^{s-3 / 4, s^{\prime}-3 / 4}$ and so we gain additional decay $\gamma^{-1 / 4} T^{1 / 8}$

$$
\left\|\mathcal{A}_{\gamma}\left(E^{2}\right)_{\check{\gamma}} \bar{E}_{\gtrsim \gamma}\right\|_{L^{2} G^{s-1, s^{\prime}-1}} \lesssim \gamma^{-1 / 4} T^{3 / 8}\left\|E_{\gtrsim \gamma}\right\|_{L^{\infty}\left(\gamma^{s-s^{\prime}} H^{s}\right)}^{2}\|E\|_{L^{8} L^{4}} .
$$

Using the interpolation (4.2), we obtain the desired bound.

\section{$6 \quad$ Energy estimate at the resonant frequency}

This estimate applies only to the true nonlinear solutions, in contrast with those in the previous section, which applies to the Duhamel formula. We denote the solution by $\mathbb{E}$ to emphasize the difference from the general functions $E$ in the previous section.

Proposition 6.1. If $\mathbb{E}$ is a solution of (1.2) such that $\mathbb{E} \in L^{\infty}\left(0, T ; G^{s, s^{\prime}}\right), \mathbb{E}_{\hat{X}} \in$ $L^{2} G_{6}^{s, s^{\prime}}(0, T)$ and $\mathbb{E} \in \mathcal{X}$ (after extending to $\mathbb{R}$ by (2.15) if necessary), then we have the following estimate

$$
\begin{aligned}
{\left[\left\|\mathbb{E}_{\hat{\gamma}}(t)\right\|_{H^{s^{\prime}}}^{2}\right]_{0}^{T} } & \lesssim\left\|\mathbb{E}_{\hat{\gamma}}\right\|_{L^{\infty} H^{s^{\prime}}}\left\{\|\mathbb{E}\|_{L^{4} L^{6}}+T^{1 / 4}\|\mathbb{E}\|_{L^{\infty} G^{s, s^{\prime}}}+T^{1 / 4}\|\mathbb{E}\|_{\mathcal{X}}\right\}^{2} \\
& \times\left\{\left\|\mathbb{E}_{\gtrsim_{\gamma}}\right\|_{L^{\infty} G^{s, s^{\prime}}}+\left\|\mathbb{E}_{\hat{X} \gtrsim \gamma}\right\|_{L^{2} G_{6}^{s, s^{\prime}}}+\left\|\mathbb{E}_{\gtrsim \gamma}\right\|_{\mathcal{X}}\right\} .
\end{aligned}
$$


Proof. Here we use the frequency localized version of the "mass decreasing" identity

$$
\partial_{t}\|\mathbb{E}\|_{L^{2}}^{2}=-\frac{\gamma \pi}{4}\left\|\mathbb{E} \cdot \mathbb{E}^{\perp}\right\|_{L^{2}(|\xi|=\gamma)}^{2},
$$

which follows from the equation. The right hand side represents the dissipation driven by nonlinear resonance at the frequency $\gamma$.

By inserting the frequency localizer at $\gamma$ in the above identity, we get

$$
\frac{1}{2} \partial_{t}\left\langle\mathbb{E} \mid \mathbb{E}_{\hat{\gamma}}\right\rangle_{x}=\Im\left\langle|\mathbb{E}|^{2} \mid \mathbb{E}_{\hat{\gamma}} \cdot \overline{\mathbb{E}}\right\rangle_{x}+\frac{1}{4} \Im\left\langle\mathcal{A}_{\gamma}\left(\mathbb{E} \cdot \mathbb{E}^{\perp}\right) \mid \mathbb{E}_{\hat{\gamma}} \cdot \mathbb{E}^{\perp}\right\rangle_{x}
$$

The first term equals

$$
\Im\left\langle\left|\mathbb{E}_{\hat{X}}+\mathbb{E}_{\hat{\gamma}}\right|^{2} \mid \mathbb{E}_{\hat{\gamma}} \cdot \overline{\mathbb{E}}_{\hat{X}}\right\rangle_{x}=\Im\left\langle\left(\left|\mathbb{E}_{\hat{X}}\right|^{2}+\left|\mathbb{E}_{\hat{\gamma}}\right|^{2}+\overline{\mathbb{E}}_{\hat{\gamma}} \cdot \mathbb{E}_{\hat{X}}\left|\mathbb{E}_{\hat{\gamma}} \cdot \overline{\mathbb{E}}_{\hat{X}}\right\rangle_{x}\right.\right.
$$

and the second term

$$
\begin{aligned}
& \Im\left\langle\mathcal{A}_{\gamma}\left(\mathbb{E}_{\hat{\gamma}}^{\perp} \cdot \mathbb{E}_{\hat{X}}\right) \mid \mathbb{E}_{\hat{\gamma}} \cdot \mathbb{E}_{\hat{\gamma}}^{\perp}\right\rangle_{x}+\Im\left\langle\mathcal{A}_{\gamma}\left(\mathbb{E}_{\hat{X}}^{\perp} \cdot \mathbb{E}_{\hat{X}}\right) \mid \mathbb{E}_{\hat{\gamma}} \cdot \mathbb{E}^{\perp}\right\rangle_{x} \\
& -\frac{\gamma \pi}{4}\left\|\mathbb{E}_{\hat{\gamma}} \cdot \mathbb{E}^{\perp}\right\|_{L^{2}(|\xi|=\gamma)}^{2}-\frac{\gamma \pi}{4}\left\|\mathbb{E}_{\hat{\gamma}} \cdot \mathbb{E}_{\hat{X}}^{\perp}\right\|_{L^{2}(|\xi|=\gamma)}^{2} .
\end{aligned}
$$

The main observation here is that we will be able to estimate all the terms appearing on (6.4) and (6.5), because of their nonresonance property, except the last two terms of (6.5), which can be neglected thanks to the good sign.

Now, we estimate the other quartic terms after integrating in time. In the term with three $\mathbb{E}_{\hat{X}}$ in (6.4), at least one of them has to have frequency $\gtrsim \gamma$. We put it in $L^{2} G_{6}^{s, s^{\prime}}$ and the two other $\mathbb{E}_{\hat{X}}$ in $L^{4} L^{6}$. Hence,

$$
\left\langle\left|\mathbb{E}_{\hat{X}}\right|^{2} \mid \mathbb{E}_{\hat{\gamma}} \cdot \mathbb{E}_{\hat{X}}\right\rangle_{t, x} \lesssim \gamma^{-2 s^{\prime}}\left\|\mathbb{E}_{\hat{\gamma}}\right\|_{L^{\infty} H^{s^{\prime}}}\left\|\mathbb{E}_{\hat{X} \gtrsim \gamma}\right\|_{L^{2} G_{6}^{s, s^{\prime}}}\left\|\mathbb{E}_{\hat{X}}\right\|_{L^{4} L^{6}}^{2}
$$

By (4.2), the right hand side is bounded by that in (6.1). The same estimate applies to the other terms in (6.4) with $\mathbb{E}_{X \gtrsim \gamma}$, so we may replace $\mathbb{E}_{\hat{X}}$ in them by $\mathbb{E}_{\ll \gamma}$.

To control them and similar terms in (6.5), we need the nonresonant property stated in the following lemma. We point out that this lemma is not needed if $s>1 / 2$ and $s^{\prime} \geq 2$. Indeed, in that case we can estimate $\mathbb{E}_{\hat{X}}$ in $L^{2} L^{\infty}$ by the Strichartz and we get better decay for the frequency of order $\gamma$.

For $\delta>0$, we decompose each function $E(t, x)$ with respect to the distance from the characteristic $\tau=|\xi|^{2} / 2$ as follows. Recall that our solution to (2.15) is defined on the whole space-time.

$$
E^{C}=\mathcal{F}_{4}^{-1} \chi\left(\left.|\tau-| \xi\right|^{2} / 2 \mid / \delta\right) \mathcal{F}_{4} E \quad E^{F}=E-E^{C},
$$

where $\chi$ is the same cut-off function as in (2.3). Also, to simplify notation, we will denote $\bar{E}^{C}=\overline{E^{C}}$. In addition, for any interval $I \in \mathbb{R}$, we denote the characteristic function of $I$ by $I$ itself and define

$$
I^{C}=\mathcal{F}_{4}^{-1} \chi(|\tau| / \delta) \mathcal{F}_{4} I \quad I^{F}=I-I^{C} .
$$


Lemma 6.2. There exists $\varepsilon>0$ small such that for $\delta<\varepsilon \gamma^{2}$ we have

$$
\begin{aligned}
0 & =\left\langle\mathbb{E}_{\ll \gamma}^{C} \mathbb{E}_{\ll \gamma}^{C} \mid \mathbb{E}_{\hat{\gamma}}^{C} \mathbb{E}_{\hat{\gamma}}^{C}\right\rangle_{t, x}=\left\langle\mathbb{E}_{\ll \gamma}^{C} \mathbb{E}_{\ll \gamma}^{C} \mid \mathbb{E}_{\hat{\gamma}}^{C} \mathbb{E}_{\hat{\gamma}}^{C} I^{C}\right\rangle_{t, x} \\
0 & =\left\langle\mathbb{E}_{\ll \gamma}^{C} \mathbb{E}_{\hat{\gamma}}^{C} \mid \mathbb{E}_{\hat{\gamma}}^{C} \mathbb{E}_{\hat{\gamma}}^{C}\right\rangle_{t, x}=\left\langle\mathbb{E}_{\ll \gamma}^{C} \mathbb{E}_{\hat{\gamma}}^{C} \mid \mathbb{E}_{\hat{\gamma}}^{C} \mathbb{E}_{\hat{\gamma}}^{C} I^{C}\right\rangle_{t, x} \\
0 & =\left\langle\mathcal{A}_{\gamma}\left(\mathbb{E}_{\ll \gamma}^{C} \mathbb{E}_{\ll \gamma}^{C}\right) \mid \mathbb{E}_{\hat{\gamma}}^{C} \mathbb{E}_{\hat{\gamma}}^{C}\right\rangle_{t, x}=\left\langle\mathcal{A}_{\gamma}\left(\mathbb{E}_{\ll \gamma}^{C} \mathbb{E}_{\ll \gamma}^{C}\right) \mid \mathbb{E}_{\hat{\gamma}}^{C} \mathbb{E}_{\hat{\gamma}}^{C} I^{C}\right\rangle_{t, x}, \\
0 & =\left\langle\mathcal{A}_{\gamma}\left(\mathbb{E}_{\ll \gamma}^{C} \mathbb{E}_{\hat{\gamma}}^{C}\right) \mid \mathbb{E}_{\hat{\gamma}}^{C} \mathbb{E}_{\hat{\gamma}}^{C}\right\rangle_{t, x}=\left\langle\mathcal{A}_{\gamma}\left(\mathbb{E}_{\ll \gamma}^{C} \mathbb{E}_{\hat{\gamma}}^{C}\right) \mid \mathbb{E}_{\hat{\gamma}}^{C} \mathbb{E}_{\hat{\gamma}}^{C} I^{C}\right\rangle_{t, x} .
\end{aligned}
$$

Proof. We only consider the version without $\mathcal{A}_{\gamma}$ and $I$, since the others are proved in the same way. Those space-time integrals are given in the following form by using the Plancherel identity,

$$
\int F_{0}\left(\tau_{0}, \xi_{0}\right) F_{1}\left(\tau_{1}, \xi_{1}\right) \overline{F_{2}}\left(\tau_{2}, \xi_{2}\right) \overline{F_{3}}\left(\tau_{3}, \xi_{3}\right) d \tau_{0} d \xi_{0} d \tau_{2} d \xi_{2} d \tau d \xi
$$

where $\left(\tau_{1}, \xi_{1}\right)$ and $\left(\tau_{3}, \xi_{3}\right)$ are such that

$$
\left(\tau_{0}, \xi_{0}\right)+\left(\tau_{1}, \xi_{1}\right)=\left(\tau_{2}, \xi_{2}\right)+\left(\tau_{3}, \xi_{3}\right)=(\tau, \xi)
$$

and each $F_{j}$ comes from some cut-offs of $\mathcal{F}_{4} \mathbb{E}$, supported in

$$
\begin{aligned}
& \left|\xi_{0}\right| \ll \gamma, \quad\left|\xi_{1}\right| \leq\left(1+\theta_{1}\right) \gamma, \quad \min \left(\left|\xi_{2}\right|,\left|\xi_{3}\right|\right) \geq\left(1-\theta_{2}\right) \gamma \\
& \left|2 \tau_{0}-\right| \xi_{0}|| \lesssim \varepsilon \gamma^{2}, \quad\left|2 \tau_{1}-\right| \xi_{1}|| \lesssim \varepsilon \gamma^{2} \\
& \left|2 \tau_{2}-\right| \xi_{2}|| \lesssim \varepsilon \gamma^{2}, \quad\left|2 \tau_{3}-\right| \xi_{3}|| \lesssim \varepsilon \gamma^{2}
\end{aligned}
$$

for some small $\theta_{1}, \theta_{2}>0$ determined by the support of $\hat{\phi}$ in Definition 2.1.

To prove that the integral vanishes, we will prove that the intersection of those supports is empty. Indeed, if $\left(\tau_{0}, \xi_{0}\right),\left(\tau_{1}, \xi_{1}\right),\left(\tau_{2}, \xi_{2}\right),\left(\tau_{3}, \xi_{3}\right)$ are all in the supports, then we have

$$
\begin{aligned}
0= & \left|\tau_{2}+\tau_{3}-\tau_{0}-\tau_{1}\right| \\
\geq & \left|\xi_{2}\right|^{2}+\left|\xi_{3}\right|^{2}-\left|\xi_{0}\right|^{2}-\left|\xi_{1}\right|^{2} \\
& -\left.\left|2 \tau_{0}-\right| \xi_{0}\right|^{2}|-| 2 \tau_{1}-\left|\xi_{1}\right|^{2}|-| 2 \tau_{2}-\left|\xi_{2}\right|^{2}|-| 2 \tau_{3}-\left|\xi_{3}\right|^{2} \mid \\
\geq & 2\left(1-\theta_{2}\right)^{2} \gamma^{2}-\left(1+\theta_{1}\right)^{2} \gamma^{2}-o\left(\gamma^{2}\right),
\end{aligned}
$$

for $\varepsilon>0$ small enough. Now the support property (S3) in Definition 2.1 implies that

$$
2\left(1-\theta_{2}\right)^{2}>\left(1+\theta_{1}\right)^{2}
$$

which gives a contradiction (for appropriate choice of $\varepsilon$ and $E_{\ll \gamma}$ in (2.5)), meaning that the support intersection is empty. 
Now, we apply the above lemma to the terms appearing in (6.4). For the second term in (6.4) (after disposing of the part $\mathbb{E}_{X \gtrsim \gamma}$ ), we have

$$
\begin{aligned}
\left\langle\left|\mathbb{E}_{\hat{\gamma}}\right|^{2} \mid \mathbb{E}_{\hat{\gamma}} \cdot \overline{\mathbb{E}}_{\ll \gamma} I\right\rangle_{t, x}= & \left\langle\mathbb{E}_{\ll \gamma}^{F} \mathbb{E}_{\hat{\gamma}} \mid \mathbb{E}_{\hat{\gamma}} \cdot \mathbb{E}_{\hat{\gamma}} I\right\rangle_{t, x} \\
& +\left\langle\mathbb{E}_{\ll \gamma}^{C} \mathbb{E}_{\hat{\gamma}}^{F} \mid \mathbb{E}_{\hat{\gamma}} \cdot \mathbb{E}_{\hat{\gamma}} I\right\rangle_{t, x} \\
& +\left\langle\mathbb{E}_{\ll \gamma}^{C} \mathbb{E}_{\hat{\gamma}}^{C} \mid \mathbb{E}_{\hat{\gamma}}^{F} \cdot \mathbb{E}_{\hat{\gamma}} I\right\rangle_{t, x} \\
& +\left\langle\mathbb{E}_{\ll \gamma}^{C} \mathbb{E}_{\hat{\gamma}}^{C} \mid \mathbb{E}_{\hat{\gamma}}^{C} \cdot \mathbb{E}_{\hat{\gamma}}^{F} I\right\rangle_{t, x} \\
& +\left\langle\mathbb{E}_{\ll \gamma}^{C} \mathbb{E}_{\hat{\gamma}}^{C} \mid \mathbb{E}_{\hat{\gamma}}^{C} \cdot \mathbb{E}_{\hat{\gamma}}^{C} I^{F}\right\rangle_{t, x},
\end{aligned}
$$

where $I=\left[0, T^{\prime}\right]$ for $T^{\prime} \in(0, T)$. The second, the third, and the fourth terms are estimated by using that

$$
\left\|\mathbb{E}_{\hat{\gamma}}^{F}\right\|_{L^{2} L^{2}} \lesssim \gamma^{-2} \gamma^{3 / 2-s^{\prime}}\left\|\mathbb{E}_{\hat{\gamma}}\right\|_{\mathcal{X}}
$$

Then they are bounded by

$$
\gamma^{3 / 2-3 s^{\prime}} T^{1 / 4}\left\|\mathbb{E}_{\hat{\gamma}}\right\|_{\mathcal{X}}\left\|\mathbb{E}_{\hat{\gamma}}\right\|_{L^{\infty} H^{s^{\prime}}}^{2}\left\|\mathbb{E}_{\ll \gamma}\right\|_{L^{4} L^{6}}
$$

For the first term of (6.15), we use

$$
\left\|\mathbb{E}_{\ll \gamma}^{F}\right\|_{L^{2} L^{2}} \lesssim \gamma^{-2} \gamma^{1-s}\|\mathbb{E}\|_{\mathcal{X}}
$$

Hence it is bounded by

$$
\gamma^{3 / 2-3 s^{\prime}} T^{1 / 2}\|\mathbb{E}\|_{\mathcal{X}}\left\|\mathbb{E}_{\hat{\gamma}}\right\|_{L^{\infty} H^{s^{\prime}}}^{3}
$$

The last term of (6.15) is bounded by

$$
\gamma^{5 / 2-3 s^{\prime}}\left\|\mathbb{E}_{\hat{\gamma}}\right\|_{L^{\infty} H^{s^{\prime}}}^{3}\|\mathbb{E}\|_{L^{\infty} G^{s, s^{\prime}}}\left\|I^{F}\right\|_{L^{1}} \lesssim T^{1 / 2} \gamma^{3 / 2-3 s^{\prime}}\left\|\mathbb{E}_{\hat{\gamma}}\right\|_{L^{\infty} H^{s^{\prime}}}^{3}\|\mathbb{E}\|_{L^{\infty} G^{s, s^{\prime}}}
$$

where we have used that $\int\left|I^{F}\right| d t \lesssim \min \left(\gamma^{-2}, T\right)$.

Remark 6.3. For the above term $\left\langle\left|\mathbb{E}_{\hat{\gamma}}\right|^{2} \mid \mathbb{E}_{\hat{X}} \cdot \overline{\mathbb{E}}_{\hat{\gamma}}\right\rangle_{t, x}$, we could have used the fact that $\mathbb{E}_{\hat{\gamma}} \in\left(L^{2} H^{s^{\prime}}, X^{s^{\prime}-3 / 2,1}\right)_{1 / 2,1} \subset L^{2} B_{6}^{s^{\prime}-3 / 4}$. However, this observation does not simplify the proof for the term $\left\langle\mathbb{E}_{\hat{X}} \cdot \overline{\mathbb{E}}_{\hat{\gamma}} \mid \mathbb{E}_{\hat{X}} \cdot \overline{\mathbb{E}}_{\hat{\gamma}}\right\rangle_{t, x}$, and moreover it is far from sufficient for the corresponding terms with the singular operator in (6.5).

Now, we estimate the last term in (6.4), namely $\left\langle\mathbb{E}_{\ll \gamma}^{2} \mid \mathbb{E}_{\hat{\gamma}}^{2}\right\rangle_{t, x}$. If $s>1 / 2$, we only need Strichartz estimate since $\mathbb{E}_{\ll \gamma}$ would be in $L^{2} L^{\infty}$. If $s=1 / 2$, we have to use the nonresonant property and argue exactly as above. Thus it is bounded by

$$
\begin{aligned}
& \gamma^{3 / 2-3 s^{\prime}} T^{1 / 4}\left\|\mathbb{E}_{\hat{\gamma}}\right\|\left\|_{\mathcal{X}}\right\| \mathbb{E}_{\hat{\gamma}}\left\|_{L^{\infty} H^{s^{\prime}}}\right\| \mathbb{E}\left\|_{L^{\infty} G^{s, s^{\prime}}}\right\| \mathbb{E} \|_{L^{4} L^{6}} \\
& +T^{1 / 2} \gamma^{3 / 2-3 s^{\prime}}\|\mathbb{E}\|_{\mathcal{X}}\left\|\mathbb{E}_{\hat{\gamma}}\right\|_{L^{\infty} H^{s^{\prime}}}^{2}\|\mathbb{E}\|_{L^{\infty} G^{s, s^{\prime}}} \\
& +T^{1 / 2} \gamma^{3 / 2-3 s^{\prime}}\left\|\mathbb{E}_{\hat{\gamma}}\right\|_{L^{\infty} H^{s^{\prime}}}^{2}\|\mathbb{E}\|_{L^{\infty} G^{s, s^{\prime}}}^{2}
\end{aligned}
$$


For the terms in (6.5), we may replace $\mathcal{A}_{\gamma}\left(E^{2}\right)$ with $\mathcal{A}_{\gamma}\left(E^{2}\right)_{\check{\gamma}}$, since we can estimate the other part $\mathcal{A}_{\gamma}\left(E^{2}\right)_{\check{X}}$ as in the case where there is no singular operator.

We decompose the first term of (6.5) into

$$
\left\langle\mathcal{A}_{\gamma}\left(\mathbb{E}_{\hat{\gamma}}^{\perp} \cdot \mathbb{E}_{\ll \gamma}\right)_{\check{\gamma}} \mid \mathbb{E}_{\hat{\gamma}}^{\perp} \cdot \mathbb{E}_{\hat{\gamma}}\right\rangle_{t, x}+\left\langle\mathcal{A}_{\gamma}\left(\mathbb{E}_{\hat{\gamma}}^{\perp} \cdot \mathbb{E}_{\gtrsim \gamma}\right)_{\check{\gamma}} \mid \mathbb{E}_{\hat{\gamma}}^{\perp} \cdot \mathbb{E}_{\hat{\gamma}}\right\rangle_{t, x},
$$

and for the second term we used the fact that $\mathcal{A}_{\gamma} \check{\phi}(|\nabla / \gamma|)$ maps $L^{3 / 2,1}$ into $L^{\infty}$ with a norm bounded by $C \gamma^{2}$ (See [15, Lemma 4.2] for the estimate on the singular part. The order in $\gamma$ is given by scaling.) Hence

$$
\left\|\mathcal{A}_{\gamma}\left(\mathbb{E}_{\hat{\gamma}}^{\perp} \cdot \mathbb{E}_{\gtrsim \gamma}\right)\right\|_{L^{\infty} L^{\infty}} \lesssim \gamma^{1-2 s^{\prime}} \gamma^{2}\left\|\mathbb{E}_{\gtrsim \gamma}\right\|_{L^{\infty}\left(\gamma^{s-s^{\prime}} H^{s}\right)}^{2}
$$

and hence the second term in (6.22) is bounded by $\gamma^{3-4 s^{\prime}} T\left\|\mathbb{E}_{\gtrsim \gamma}\right\|_{L^{\infty}\left(\gamma^{s-s^{\prime}} H^{s}\right)}^{2}\left\|\mathbb{E}_{\hat{\gamma}}\right\|_{L^{\infty} H^{s^{\prime}}}^{2}$.

For the first term of (6.22), we use the above lemma, writing the decomposition as in (6.15). For $\left\langle\mathcal{A}_{\gamma}\left(\mathbb{E}_{\hat{\gamma}}^{F \perp} \cdot \mathbb{E}_{\ll \gamma}\right) \check{\gamma} \mid \mathbb{E}_{\hat{\gamma}}^{\perp} \cdot \mathbb{E}_{\hat{\gamma}} I\right\rangle_{t, x}$, we use that

$$
\left\|\mathbb{E}_{\hat{\gamma}}^{F \perp} \cdot \mathbb{E}_{\ll \gamma}\right\|_{L^{4 / 3} L^{3 / 2,1}} \lesssim\left\|\mathbb{E}_{\hat{\gamma}}^{F}\right\|_{L^{2} L^{2}}\left\|\mathbb{E}_{\ll \gamma}\right\|_{L^{4} L^{6,2}} .
$$

Then, using that $\mathcal{A}_{\gamma} \check{\phi}(|\nabla / \gamma|)$ maps $L^{3 / 2,1}$ into $L^{\infty}$ with a norm bounded by $C \gamma^{2}$, we can dominate this term by

$$
T^{1 / 4} \gamma^{-2 s^{\prime}}\|\mathbb{E}\|_{\mathcal{X}}\left\|\mathbb{E}_{\hat{X}}\right\|_{L^{4} L^{6,2}}\left\|\mathbb{E}_{\hat{\gamma}}\right\|_{L^{\infty} H^{s^{\prime}}}^{2}
$$

Using (4.2), we can bound the right side by that in (6.1).

For $\left\langle\mathcal{A}_{\gamma}\left(\mathbb{E}_{\hat{\gamma}}^{\perp} \cdot \mathbb{E}_{\ll \gamma}^{F}\right)_{\check{\gamma}} \mid \mathbb{E}_{\hat{\gamma}}^{\perp} \cdot \mathbb{E}_{\hat{\gamma}} I\right\rangle_{t, x}$, we use that

$$
\left\|\mathbb{E}_{\hat{\gamma}}^{\perp} \cdot \mathbb{E}_{\ll \gamma}^{F}\right\|_{L^{2} L^{6 / 5}} \lesssim \gamma^{-s^{\prime}} \gamma^{1 / 2} \gamma^{-3 / 2}\left\|\mathbb{E}_{\hat{\gamma}}\right\|_{L^{\infty} H^{s^{\prime}}}\|\mathbb{E}\|_{\mathcal{X}}
$$

and that $\mathcal{A}_{\gamma} \check{\phi}(|\nabla / \gamma|)$ maps $L^{6 / 5}$ into $L^{6}$ with a norm bounded by $\gamma^{2}$ (cf. [15, Lemma $4.2])$. Hence this term is bounded by

$$
T^{1 / 2} \gamma^{3 / 2-s^{\prime}} \gamma^{-2 s^{\prime}}\left\|\mathbb{E}_{\hat{\gamma}}\right\|_{L^{\infty} H^{s^{\prime}}}^{3}\|\mathbb{E}\|_{\mathcal{X}}
$$

The estimate of the other three terms are the same and we do not detail them. We get

$$
\begin{aligned}
& \left|\left\langle\mathcal{A}_{\gamma}\left(\mathbb{E}_{\hat{\gamma}}^{C \perp} \cdot \mathbb{E}_{\ll \gamma}^{C}\right)_{\check{\gamma}} \mid \mathbb{E}_{\hat{\gamma}}^{F \perp} \cdot \mathbb{E}_{\hat{\gamma}} I\right\rangle_{t, x}\right| \lesssim T^{1 / 4} \gamma^{-2 s^{\prime}}\|\mathbb{E}\|_{\mathcal{X}}\left\|\mathbb{E}_{\hat{X}}\right\|_{L^{4} L^{6,2}}\left\|\mathbb{E}_{\hat{\gamma}}\right\|_{L^{\infty} H^{s^{\prime}}}^{2}, \\
& \left|\left\langle\mathcal{A}_{\gamma}\left(\mathbb{E}_{\hat{\gamma}}^{C \perp} \cdot \mathbb{E}_{\ll \gamma}^{C}\right)_{\check{\gamma}} \mid \mathbb{E}_{\hat{\gamma}}^{C \perp} \cdot \mathbb{E}_{\hat{\gamma}}^{F} I\right\rangle_{t, x}\right| \lesssim T^{1 / 4} \gamma^{-2 s^{\prime}}\|\mathbb{E}\|_{\mathcal{X}}\left\|\mathbb{E}_{\hat{X}}\right\|_{L^{4} L^{6,2}}\left\|\mathbb{E}_{\hat{\gamma}}\right\|_{L^{\infty} H^{s^{\prime}}}^{2}, \\
& \left|\left\langle\mathcal{A}_{\gamma}\left(\mathbb{E}_{\hat{\gamma}}^{C \perp} \cdot \mathbb{E}_{\ll \gamma}^{C}\right)_{\check{\gamma}} \mid \mathbb{E}_{\hat{\gamma}}^{C \perp} \cdot \mathbb{E}_{\hat{\gamma}}^{C} I^{F}\right\rangle_{t, x}\right| \lesssim T^{1 / 2} \gamma^{3 / 2-3 s^{\prime}}\left\|\mathbb{E}_{L^{\infty} G^{s, s^{\prime}}}\right\| \mathbb{E}_{\hat{\gamma}} \|_{L^{\infty} H^{s^{\prime}}}^{3} .
\end{aligned}
$$

Finally, we treat the second term in (6.5). Here, we can not use the nonresonant property. However, by the Fourier support property (S2) in Definition 2.1, we have

$$
\left(\mathbb{E}_{\hat{X}}^{\perp} \cdot \mathbb{E}_{\ll \gamma}\right)_{\check{\gamma}}=0=\left(\mathbb{E}_{\ll \gamma}^{\perp} \cdot \mathbb{E}_{\hat{X}}\right)_{\check{\gamma}},
$$


hence by using Hölder,

$$
\begin{aligned}
\left\|\left(\mathbb{E}_{\hat{X}}^{\perp} \cdot \mathbb{E}_{\hat{X}}\right)_{\grave{\gamma}}\right\|_{L^{8 / 3} L^{4 / 3}} & \lesssim\left\|\mathbb{E}_{\hat{X} \gtrsim \gamma}\right\|_{L^{16 / 3} L^{8 / 3}}^{2} \\
& \lesssim \gamma^{-2 s^{\prime}}\left\|\mathbb{E}_{\gtrsim \gamma}\right\|_{L^{\infty}\left(\gamma^{s-s^{\prime}} H^{s}\right)}^{5 / 4}\left\|\mathbb{E}_{\hat{X} \gtrsim \gamma}\right\|_{L^{2} G_{6}^{s, s^{\prime}}}^{3 / 4}
\end{aligned}
$$

and so by using the $L^{4 / 3} \rightarrow L^{4}$ bound on $\mathcal{A}_{\gamma}$,

$$
\left\|\mathcal{A}_{\gamma}\left(\mathbb{E}_{\hat{X}}^{+} \cdot \mathbb{E}_{\hat{X}}\right)\right\|_{\gamma}\left\|_{L^{8 / 3} L^{4}} \lesssim \gamma^{3 / 2-2 s^{\prime}}\right\| \mathbb{E}_{\gtrsim \gamma}\left\|_{L^{\infty}\left(\gamma^{s-s^{\prime}} H^{s}\right)}^{5 / 4}\right\| \mathbb{E}_{\hat{X} \gtrsim \gamma} \|_{L^{2} G_{6}^{s, s^{\prime}}}^{3 / 4}
$$

Then, we put the last $\mathbb{E}_{\hat{X}}$ in $L^{8} L^{4}$ by (4.2) (notice that this part may have low frequency), and the term is controlled by

$$
T^{1 / 2} \gamma^{3 / 2-3 s^{\prime}}\left\|\mathbb{E}_{\hat{\gamma}}\right\|_{L^{\infty} H^{s^{\prime}}}\left\|\mathbb{E}_{\gtrsim \gamma}\right\|_{L^{\infty}\left(\gamma^{\left.s-s^{\prime} H^{s}\right)}\right.}^{5 / 4}\left\|\mathbb{E}_{\hat{X} \gtrsim \gamma}\right\|_{L^{2} G_{6}^{s, s^{\prime}}}^{3 / 4}\left\|\mathbb{E}_{\hat{X}}\right\|_{L^{8} L^{4}}
$$

which is dominated by the right side of (6.1) due to (4.2).

\subsection{Uniform estimate}

For the global solution $\mathbb{E}$ of $(2.15)$ for any fixed $T \in(0,1)$, we denote

$$
G=\|\mathbb{E}\|_{L^{\infty} G^{s, s^{\prime}}}, \quad S=\left\|\mathbb{E}_{\hat{X}}\right\|_{L^{2} G_{6}^{s, s^{\prime}}}, \quad X=\|\mathbb{E}\|_{\mathcal{X}}, \quad L=\|\mathbb{E}\|_{L^{4} L^{6}}
$$

Notice that by definition of the integral equation (2.15), those $L_{t}^{p}$ norms on $t \in \mathbb{R}$ are dominated by their restriction to $[0, T]$. We also denote the initial data size by

$$
G(0):=\|\mathbb{E}(0)\|_{G^{s, s^{\prime}}}, \quad L(0):=\left\|e^{-i t \Delta / 2} \mathbb{E}(0)\right\|_{L^{4} L^{6}(0, T)} .
$$

Note that $L(0) \rightarrow 0$ as $T \rightarrow+0$ uniformly for $\gamma \rightarrow \infty$, because $\mathbb{E}(0) \rightarrow \mathbb{E}^{\infty}(0)$ in $H^{s}$. This is essential in the scaling critical case $s=1 / 2$.

Putting the results of Propositions 4.3, 5.1 and 6.1 together, and using that $\left[L^{\infty} G^{s, s^{\prime}}, L^{2} G_{6}^{s, s^{\prime}}\right]_{1 / 2} \subset L^{4} L^{6}$, we deduce that

$$
\begin{aligned}
& L \leq C\left\{L(0)+\left[L+T^{1 / 4} G\right]^{2} G\right\}, \\
& S+X \leq C\left\{G(0)+\left[L+T^{1 / 4} G\right]^{2} G\right\}, \\
& G \leq C\left\{G(0)+\left[L+T^{1 / 4} G+(T+1 / \gamma)^{1 / 4} X\right]^{2}(G+S)\right\},
\end{aligned}
$$

for some absolute constant $C \geq 1$.

Take $M \geq 2(C+2)$. Then for small $T>0$ we have

$$
8 M^{3} C\left[L(0)+T^{1 / 4} G(0)+(T+1 / \gamma)^{1 / 4} G(0)\right](G(0)+1) \leq 1 / 2 .
$$


More precisely, there exists $T_{0}>0$ such that if $T \leq T_{0}$ then the above condition holds. Note that $T_{0}$ is independent of $\gamma$, because $L(0) \rightarrow 0$ as $T \rightarrow+0$ uniformly in $\gamma$, and $G(0)$ converges as $\gamma \rightarrow \infty$.

We may assume that $G(0) \neq 0$ since otherwise the solution is trivial. Suppose that we have

$$
L \leq M\left[L(0)+T^{1 / 4} G(0)\right], \quad G \leq M G(0)
$$

Then from the above inequalities we obtain

$$
\begin{aligned}
L & \leq C L(0)+4 M^{3} C\left[L(0)+T^{1 / 4} G(0)\right]^{2} G(0) \\
& \leq(C+1)\left[L(0)+T^{1 / 4} G(0)\right]<\frac{M}{2}\left[L(0)+T^{1 / 4} G(0)\right], \\
S+X & \leq C G(0)+4 M^{3} C\left[L(0)+T^{1 / 4} G(0)\right]^{2} G(0) \\
& \leq(C+1) G(0)<\frac{M}{2} G(0),
\end{aligned}
$$

hence in the same way we get

$$
\begin{aligned}
G & \leq C G(0)+8 M^{3} C\left[L(0)+T^{1 / 4} G(0)+(T+1 / \gamma)^{1 / 4} G(0)\right]^{2} G(0) \\
& \leq(C+1) G(0)<\frac{M}{2} G(0) .
\end{aligned}
$$

Since (6.37) holds at least for sufficiently small $T>0$ (it may depend on $\gamma$ ), and $L$ and $G$ are continuous with respect to $T$, we have (6.37) as long as (6.36) is satisfied. Thus we have

$$
\|\mathbb{E}\|_{L^{\infty} G^{s, s^{\prime}}(0, T)}+\|\mathbb{E}\|_{L^{2} G_{6}^{s, s^{\prime}}(0, T)}+\|\mathbb{E}\|_{\mathcal{X}} \leq M\|\mathbb{E}(0)\|_{G^{s, s^{\prime}}}
$$

for $T \leq T_{0}$.

It is classical to construct solutions on a fixed time interval using the above uniform a priori estimates and we do not detail this here.

\section{Convergence proof}

For the convergence proof, we use the Duhamel formula for the nonresonant part and write

$$
\begin{aligned}
\mathbb{E}-\mathbb{E}^{\infty}= & e^{-i \frac{t}{2} \Delta}\left(\mathbb{E}^{\gamma}(0)-\mathbb{E}^{\infty}(0)\right) \\
& +S_{T}\left[\left(|\mathbb{E}|^{2} \mathbb{E}-\left|\mathbb{E}^{\infty}\right|^{2} \mathbb{E}^{\infty}\right)+\mathcal{A}_{\gamma}\left(\mathbb{E} \cdot \mathbb{E}^{\perp}-\mathbb{E}^{\infty} \cdot \mathbb{E}^{\infty \perp}\right) \overline{\mathbb{E}}\right] \\
& +S_{T}\left[\mathcal{A}_{\gamma}\left(\mathbb{E}^{\infty} \cdot \mathbb{E}^{\infty \perp}\right) \overline{\mathbb{E}}\right] .
\end{aligned}
$$


The Strichartz estimate implies

$$
\begin{aligned}
& \left\|\left(\mathbb{E}-\mathbb{E}^{\infty}\right)_{\hat{X}}\right\|_{L^{\infty} H^{s} \cap L^{2} B_{6}^{s}} \lesssim\left\|\left(\mathbb{E}-\mathbb{E}^{\infty}\right)(0)\right\|_{H^{s}}+I_{1}+I_{2}+I_{3}, \\
& I_{1}:=\left\||\mathbb{E}|^{2} \mathbb{E}-\left|\mathbb{E}^{\infty}\right|^{2} \mathbb{E}^{\infty}\right\|_{L^{2} B_{6 / 5}^{s}}+\left\|\mathcal{A}_{\gamma}\left(\mathbb{E} \cdot \mathbb{E}^{\perp}-\mathbb{E}^{\infty} \cdot \mathbb{E}^{\infty \perp}\right)_{\check{X}} \overline{\mathbb{E}}\right\|_{L^{2} B_{6 / 5}^{s}}, \\
& I_{2}:=\left\|\left[\mathcal{A}_{\gamma}\left(\mathbb{E} \cdot \mathbb{E}^{\perp}\right)_{\check{\gamma}} \overline{\mathbb{E}}\right]_{\hat{X}}\right\|_{L^{8 / 5} B_{4 / 3}^{s}}, \\
& I_{3}:=\left\|\mathcal{A}_{\gamma}\left(\mathbb{E}^{\infty} \cdot \mathbb{E}^{\infty \perp}\right)_{\check{X}} \overline{\mathbb{E}}\right\|_{L^{2} B_{6 / 5}^{s}} .
\end{aligned}
$$

Estimating in the same way as in (4.4), we obtain

$$
\begin{aligned}
I_{1} \lesssim & \left\|\mathbb{E}-\mathbb{E}^{\infty}\right\|_{L^{\infty} H^{s} \cap L^{4} L^{6}}\left\||\mathbb{E}|+\left|\mathbb{E}^{\infty}\right|\right\|_{L^{4} L^{6}} \\
& \times\left\{\|\mathbb{E}\|_{L^{\infty} H^{s} \cap L^{4} L^{6}}+\left\|\mathbb{E}^{\infty}\right\|_{L^{\infty} H^{s} \cap L^{4} L^{6}}\right\} \\
I_{2} \lesssim & T^{1 / 2} \gamma^{s-s^{\prime}}\left\|\mathbb{E}_{\gtrsim \gamma}\right\|_{L^{\infty}\left(\gamma^{\left.s-s^{\prime} H^{s}\right)}\right.}^{2}\|\mathbb{E}\|_{L^{8} L^{4}} .
\end{aligned}
$$

In addition, we have

$$
I_{3} \rightarrow 0
$$

as $\gamma \rightarrow \infty$, by approximating $\mathbb{E}^{\infty}$ by $C^{\infty}$ functions.

If $s<s^{\prime}$, then $I_{2} \rightarrow 0$, and $\|\mathbb{E}\|_{L^{4} L^{6}(0, T)}+\left\|\mathbb{E}^{\infty}\right\|_{L^{4} L^{6}(0, T)} \ll 1$ if $T>0$ is small enough. Since the $L^{4} L^{6}$ norm is dominated by that of $L^{\infty} H^{s} \cap L^{2} B_{6}^{s}$, and moreover vanishing as $T \rightarrow+0$ uniformly in $\gamma$, we obtain

$$
\left\|\left(\mathbb{E}-\mathbb{E}^{\infty}\right)_{\hat{X}}\right\|_{L^{\infty} H^{s} \cap L^{2} B_{6}^{s}} \ll\left\|\mathbb{E}-\mathbb{E}^{\infty}\right\|_{L^{\infty} H^{s} \cap L^{2} B_{6}^{s}}+o(1),
$$

for $T>0$ small, where $o(1) \rightarrow 0$ as $\gamma \rightarrow \infty$ uniformly in $T \in(0,1)$.

In the case $s=s^{\prime} \geq 3 / 2, I_{2}$ does not have explicit decay factor, so we need to approximate $\mathbb{E}$ by $\mathbb{E}^{\infty}$ also in $I_{2}$. Then we have in the same way as (4.4),

$$
\begin{aligned}
& \left\|\left[\mathcal{A}_{\gamma}\left(\mathbb{E} \cdot \mathbb{E}^{\perp}-\mathbb{E}^{\infty} \cdot \mathbb{E}^{\infty \perp}\right)_{\check{\gamma}} \overline{\mathbb{E}}\right]_{\hat{X}}\right\|_{L^{8 / 5} B_{4 / 3}^{s}} \\
& \quad \lesssim T^{1 / 2}\|\mathbb{E}\|_{L^{\infty} H^{s}}\left\|\mathbb{E}-\mathbb{E}^{\infty}\right\|_{L^{\infty} H^{s} \cap L^{8} L^{4}}\left\{\|\mathbb{E}\|_{L^{\infty} H^{s} \cap L^{8} L^{4}}+\left\|\mathbb{E}^{\infty}\right\|_{L^{\infty} H^{s} \cap L^{8} L^{4}}\right\} \\
& \left\|\left[\mathcal{A}_{\gamma}\left(\mathbb{E}^{\infty} \cdot \mathbb{E}^{\infty \perp}\right)_{\check{\gamma}} \overline{\mathbb{E}}\right]_{\hat{X}}\right\|_{L^{8 / 5} B_{4 / 3}^{s}} \lesssim T^{1 / 2}\|\mathbb{E}\|_{L^{\infty} H^{s}}\left\|\mathbb{E}^{\infty}\right\|_{L^{8} L^{4}}\left\|\mathbb{E}_{\gtrsim_{\gamma}}^{\infty}\right\|_{L^{\infty} H^{s}},
\end{aligned}
$$

and the last norm goes to 0 . Since the other norms are uniformly bounded, we get (7.5) for small $T>0$.

Now, we have to estimate $\left\|\left(\mathbb{E}-\mathbb{E}^{\infty}\right)_{\hat{\gamma}}\right\|_{L^{\infty} H^{s}}$. It is enough to estimate $\left\|\mathbb{E}_{\hat{\gamma}}\right\|_{L^{\infty} H^{s}}$ since $\left\|\mathbb{E}_{\hat{\gamma}}^{\infty}\right\|_{L^{\infty} H^{s}}$ vanishes. Also, if $s^{\prime}>s$, then $\left\|\mathbb{E}_{\hat{\gamma}}\right\|_{L^{\infty} H^{s}}$ goes to zero thanks to the uniform bound in $G^{s, s^{\prime}}$. Hence, we have only to deal with the case $s=s^{\prime} \geq 3 / 2$. ¿From (6.1) we have

$$
\begin{aligned}
{\left[\left\|\mathbb{E}_{\hat{\gamma}}\right\|_{H^{s}}\right]_{0}^{T} \ll } & \left\|\mathbb{E}_{\gtrsim_{\gamma}}\right\|_{L^{\infty} G^{s, s^{\prime}}}+\left\|\mathbb{E}_{\hat{X} \gtrsim \gamma}\right\|_{L^{2} G_{6}^{s, s^{\prime}}}+\left\|\mathbb{E}_{\gtrsim \gamma}\right\|_{\hat{X}} \\
& \lesssim o(1)+\left\|\mathbb{E}-\mathbb{E}^{\infty}\right\|_{L^{\infty} H^{s}}+\left\|\left(\mathbb{E}-\mathbb{E}^{\infty}\right)_{\hat{X}}\right\|_{L^{2} B_{6}^{s}}+\left\|\mathbb{E}_{\gtrsim \gamma}\right\|_{\mathcal{X}},
\end{aligned}
$$


for small $T>0$, and by the same argument for (5.1), we have also

$$
\left\|\mathbb{E}_{\gtrsim \gamma}\right\|_{\mathcal{X}} \lesssim o(1)+\left\|\mathbb{E}_{\gtrsim \gamma / 4}\right\|_{L^{\infty} G^{s, s^{\prime}}} \lesssim o(1)+\left\|\mathbb{E}-\mathbb{E}^{\infty}\right\|_{L^{\infty} H^{s}}
$$

Therefore in all cases we have

$$
\begin{aligned}
& \left\|\mathbb{E}-\mathbb{E}^{\infty}\right\|_{L^{\infty} H^{s}}+\left\|\left(\mathbb{E}-\mathbb{E}^{\infty}\right)_{\hat{X}}\right\|_{L^{2} B_{6}^{s}} \\
& \quad \ll o(1)+\left\|\mathbb{E}-\mathbb{E}^{\infty}\right\|_{L^{\infty} H^{s}}+\left\|\left(\mathbb{E}-\mathbb{E}^{\infty}\right)_{\hat{X}}\right\|_{L^{2} B_{6}^{s}},
\end{aligned}
$$

for small $T>0$, hence $\mathbb{E} \rightarrow \mathbb{E}^{\infty}$ in $L^{\infty} H^{s}(0, T)$.

We can repeat this argument from $t=T$ to prolong the interval of convergence as many times as we like. Since the smallness condition on $T$ can be determined only by

$$
\lim _{\gamma \rightarrow \infty}\left\|e^{i t \Delta / 2} \mathbb{E}(0)\right\|_{L^{4} L^{6}(0, T)}=\left\|e^{i t \Delta / 2} \mathbb{E}^{\infty}(0)\right\|_{L^{4} L^{6}(0, T)},
$$

we can extend the convergence interval as long as $\mathbb{E}^{\infty}$ stays in $L^{4} L^{6}$, i.e. up to the maximal existence time $T^{\infty}$. More precisely, for any $T^{\prime} \in\left(0, T^{\infty}\right)$ and any $\varepsilon \in(0,1)$, we can decompose $\left(0, T^{\prime}\right)$ by $0=T_{0}<T_{1}<\cdots<T_{N}=T^{\prime}$ with some $N \in \mathbb{N}$, such that for all $j=0, \ldots, N-1$,

$$
\left\|\mathbb{E}^{\infty}\right\|_{L^{4} L^{6}\left(T_{j}, T_{j+1}\right)} \leq \varepsilon
$$

Then applying the Strichartz estimate on $I_{j}:=\left(T_{j}, T_{j+1}\right)$, we get

$$
\begin{aligned}
\left\|e^{i\left(t-T_{j}\right) \Delta / 2} \mathbb{E}^{\infty}\left(T_{j}\right)\right\|_{L^{4} L^{6}\left(I_{j}\right)} & \leq\left\|\mathbb{E}^{\infty}\right\|_{L^{4} L^{6}\left(I_{j}\right)}+C\left\|\mathbb{E}^{\infty}\right\|_{L^{\infty} H^{s}\left(I_{j}\right)}\left\|\mathbb{E}^{\infty}\right\|_{L^{4} L^{6}\left(I_{j}\right)}^{2} \\
& \leq 2 \varepsilon,
\end{aligned}
$$

if $\varepsilon>0$ is sufficiently small. Making $\varepsilon>0$ small enough to satisfy the smallness condition for the convergence, we get the convergence up to $t=T^{\prime}$ by $N$ times repetition of the above argument.

\section{The limit $\gamma$ going to 0}

To prove theorem 3.3, we first prove the existence on a uniform time interval and then prove the convergence.

The proof of existence is the same as for the proof of existence for the nonlinear Schrödinger in $H^{1 / 2}$. However, due to the presence of the operator $\mathcal{A}_{\gamma}$ here, we have to choose the Strichartz spaces the right way. We have

$$
\left\|\mathbb{E} \cdot \mathbb{E}^{\perp}\right\|_{L^{8 / 3}\left(B_{4 / 3}^{s} \cap B_{12 / 7}^{s}\right)} \lesssim\|\mathbb{E}\|_{L^{\infty} H^{s}}^{5 / 4}\|\mathbb{E}\|_{L^{2} B_{6}^{s}}^{3 / 4}
$$


and hence

$$
\left\|\mathcal{A}_{\gamma}\left(\mathbb{E} \cdot \mathbb{E}^{\perp}\right)\right\|_{L^{8 / 3}\left(B_{4}^{s}+B_{12 / 7}^{s}\right)} \lesssim\|\mathbb{E}\|_{L^{\infty} H^{s}}^{5 / 4}\|\mathbb{E}\|_{L^{2} B_{6}^{s}}^{3 / 4}
$$

Then, we multiply by $\overline{\mathbb{E}}$

$$
\begin{aligned}
& \left\|\mathcal{A}_{\gamma}\left(\mathbb{E} \cdot \mathbb{E}^{\perp}\right) \overline{\mathbb{E}}\right\|_{L^{4 / 3} B_{2 / 3}^{s}} \\
& \lesssim\left\|\mathcal{A}_{\gamma}(\mathbb{E} \cdot \mathbb{E})\right\|_{L^{8 / 3}\left(B_{4}^{s}+B_{12 / 7}^{s}\right)}\left(T^{1 / 2}\|\mathbb{E}\|_{L^{8} B_{12 / 5}^{s}}+\|\mathbb{E}\|_{L^{8 / 3} B_{4}^{s}}\right) \\
& \lesssim T^{1 / 2}\|\mathbb{E}\|_{L^{\infty} H^{s}}^{2}\|\mathbb{E}\|_{L^{2} B_{6}^{s}}+\|\mathbb{E}\|_{L^{\infty} H^{s}}^{3 / 2}\|\mathbb{E}\|_{L^{2} B_{6}^{s}}^{3 / 2}
\end{aligned}
$$

Hence, if $G_{T}$ denotes $\|\mathbb{E}\|_{L^{\infty}\left(0, T ; H^{s}\right)}$ and $S_{T}$ denotes $\|\mathbb{E}\|_{L^{2} B_{6}^{s}(0, T)}$, we deduce that

$$
\begin{aligned}
G_{T} & \leq G_{0}+C\left[G_{T}^{3 / 2} S_{T}^{3 / 2}+G_{T}^{2} T^{1 / 2} S_{T}\right] \\
S_{T} & \leq\left\|e^{-i \frac{t}{2} \Delta} \mathbb{E}(0)\right\|_{L^{2} B_{6}^{s}}+C\left[G_{T}^{3 / 2} S_{T}^{3 / 2}+G_{T}^{2} T^{1 / 2} S_{T}\right] .
\end{aligned}
$$

Hence, if we choose $T$ such that

$$
\begin{gathered}
\left\|e^{-i \frac{t}{2} \Delta} \mathbb{E}(0)\right\|_{L^{2} B_{6}^{s}} \leq \frac{\varepsilon}{\left(2 G_{0}+1\right)^{3}}, \\
T \leq \frac{\varepsilon}{C\left(2 G_{0}+1\right)^{4}},
\end{gathered}
$$

we deduce by continuity of $S_{T}$ and $G_{T}$ that

$$
S_{T} \leq \frac{4 \varepsilon}{\left(2 G_{0}+1\right)^{3}}, \quad G_{T} \leq \frac{3}{2} G_{0}+1 .
$$

This insures the existence on a uniform time interval.

The convergence proof is exactly similar and much easier than the case $\gamma$ going to infinity, because

$$
\mathcal{A}_{\gamma}(\cdot)_{>2 \gamma} \rightarrow 1
$$

strongly on any Besov space as $\gamma \rightarrow 0$, and

$$
\left\|\mathcal{A}_{\gamma}\left(\mathbb{E} \cdot \mathbb{E}^{\perp}\right)_{\leq 2 \gamma}\right\|_{L^{8 / 3} B_{4}^{s}} \lesssim \gamma^{3 / 2}
$$

We do not give more details. In particular it yields the fact that when $\gamma$ goes to zero the existence time of the initial system (1.2) $T^{\gamma}$ satisfies (3.6).

\section{A Appendix: $L^{p}$ bounds on $\mathcal{A}_{\gamma}$}

In this appendix, we prove that when restricted to frequencies of order $\gamma, \mathcal{A}_{\gamma}$ is bounded from $L^{4 / 3}$ to $L^{4}$ with a norm bounded by $\gamma^{3 / 2}$. By scaling it is enough to prove it for $\gamma=1$. When $\mathcal{A}_{\gamma}$ is replaced by $\delta_{|\nabla|=1}$, then the statement is the Tomas-Stein theorem (see [21]). Here, we would like to adapt that argument to deal with $P V\left(\frac{|\nabla|^{2}}{|\nabla|^{2}-1}\right)$. More precisely, we prove the lemma 
Lemma A.1. If $\operatorname{supp} \mathcal{F} f \in\{1 / 2<|\xi|<2\}$, then

$$
\left\|P V\left(\frac{|\nabla|^{2}}{|\nabla|^{2}-1}\right)(f)\right\|_{L^{4}} \leq C\|f\|_{L^{4 / 3}} .
$$

Remark A.2. This lemma also holds in dimension $n \geq 2$, in which case $L^{4 / 3}$ is replaced by $L^{\frac{2(n+1)}{n+3}}$ and $L^{4}$ is replaced by $L^{\frac{2(n+1)}{n-1}}$

We will give the proof in dimension $n$. In the next subsection, we give an alternative proof which uses the Tomas-Stein theorem and gives a stronger conclusion.

For the proof, we follow the proof of the Tomas-Stein theorem (see the notes of Tao [21]). We denote $T=P V \frac{2 \chi(|\nabla|)}{|\nabla|^{2}-1}$ where supp $\chi \in(1 / 3,3)$ and $\chi(\xi)=1$ for $1 / 2 \leq|\xi| \leq 2$. We also denote $K(x)$ its kernel. One can prove by explicit Fourier transform

$$
|K(x)| \leq C|x|^{-\frac{n-1}{2}}
$$

Let $\phi$ be a radial smooth function which is equal to 1 near zero and which is compactly supported. We define for $k \in \mathbb{N} \psi_{k}(x)=\phi\left(2^{-k} x\right)-\phi\left(2^{1-k} x\right)$. Hence, $\psi_{k}(x)=$ $\psi_{0}\left(2^{-k} x\right)$ and $1=\phi(x)+\sum_{k \in \mathbb{N}} \psi_{k}(x)$. Hence, we can write

$$
T(f)=K * f=(\phi K) * f+\sum_{k \in \mathbb{N}}\left(\psi_{k} K\right) * f .
$$

The estimate on the first term is trivial from the kernel bound (A.2). To sum the other operators, we use the complex interpolation. Hence lemma A.1 follows from

$$
\begin{aligned}
& \sup _{t \in \mathbb{R}}\left\|\sum_{k \in \mathbb{N}} 2^{\left(\frac{n-1}{2}+i t\right) k}\left(\psi_{k} K\right) * f\right\|_{L^{\infty}} \leq C\|f\|_{L^{1}}, \\
& \sup _{t \in \mathbb{R}}\left\|\sum_{k \in \mathbb{N}} 2^{(-1+i t) k}\left(\psi_{k} K\right) * f\right\|_{L^{2}} \leq C\|f\|_{L^{2}},
\end{aligned}
$$

by interpolating them at $1 / 2$.

Hence, it remains to prove these two estimates. The first estimate follows from (A.2). For the proof of the second estimate, we have to show that

$$
\sum_{k \in \mathbb{N}} 2^{-k}\left|\widetilde{\psi_{k} K}\right| \leq C
$$

This is a consequence of the following claim

$$
\widetilde{\psi_{k} K}(\xi) \mid \leq \begin{cases}C+C 2^{k}\left(2^{k} d\right)^{-1} & d \geq 2^{-k} \\ C+C 2^{k}\left(2^{k} d\right) & d \leq 2^{-k}\end{cases}
$$

where $d=|1-| \xi||$ is the distance from $\xi$ to the unit sphere. 
First, consider the case $d \geq 2^{-k}$. Modulo a rotation, we can assume that $\xi=$ $|\xi| e_{1}$. In the following computations, we will omit $P V$. We have

$$
\begin{aligned}
\int \widetilde{\psi}_{k}(\xi-y) \frac{1}{|y|-1} d y= & \int_{|r-1|>d / 2} \widetilde{\psi}_{k}(\xi-r \omega) \frac{1}{r-1} r^{n-1} d r d \omega \\
& +\int_{|r-1|<d / 2} \frac{\widetilde{\psi}_{k}(\xi-r \omega) r^{n-1}-\widetilde{\psi}_{k}(\xi-\omega)}{r-1} d r d \omega
\end{aligned}
$$

where we put $y=r \omega$ with $\omega \in S^{n-1}$. The first term on the right hand side is bounded by $C / d$. For the second term, we have

$$
\begin{aligned}
& \int_{|r-1|<d / 2} \frac{\widetilde{\psi}_{k}(\xi-r \omega) r^{n-1}-\widetilde{\psi}_{k}(\xi-\omega)}{r-1} d r d \omega \\
= & \int_{|r-1|<d / 2} \widetilde{\psi}_{k}(\xi-r \omega) \frac{r^{n-1}-1}{r-1} d r d \omega \\
& \quad+\int_{|r-1|<d / 2} \frac{\widetilde{\psi}_{k}(\xi-r \omega)-\widetilde{\psi}_{k}(\xi-\omega)}{r-1} r^{n-1} d r d \omega
\end{aligned}
$$

The first term is bounded by a constant $C$. For the second term, we split the integral over $S^{n-1}$ into annulus where $\left|\omega-e_{1}\right|$ is of order $2^{-k+j}$ and $j \in \mathbb{N}$, replace the difference by the derivative of $\widetilde{\psi}_{k}$ and use its rapid decay. Hence, it is bounded by

$$
\sum_{j \in \mathbb{N}} \int_{\substack{|r-1|<d / 2 \\\left|\omega-e_{1}\right| \leq 2^{-k+j}}} \frac{2^{k} 2^{n k}}{\left[1+2^{k}\left(d+2^{-k+j}\right)\right]^{N}} r^{n-1} d r d \omega \lesssim \sum_{j \in \mathbb{N}} \frac{2^{k} 2^{n k} d 2^{(-k+j)(n-1)}}{\left[1+2^{k}\left(d+2^{-k+j}\right)\right]^{N}},
$$

for any $N \in \mathbb{N}$, which is bounded by

$$
\frac{2^{k} 2^{k n} d^{n}}{\left(1+2^{k} d\right)^{N}} \leq C / d
$$

Now, we consider the case where $d<2^{-k}$. First, we observe that $\left|\widetilde{\nabla \psi_{k} K}(\xi)\right| \lesssim$ $2^{2 k}$. We use that $\nabla \widetilde{\psi_{k}}(\eta)=2^{2 k} f_{k}(\eta) \eta$, where $\left|f_{k}(\eta)\right| \lesssim \frac{2^{k n}}{\left(1+2^{k}|\eta|\right)^{N}}$ and $\left|f_{k}^{\prime}(\eta)\right| \lesssim$ $\frac{2^{k} 2^{k n}}{\left(1+2^{k}|\eta|\right)^{N}}$. Hence, it is enough to bound

$$
\begin{aligned}
& 2^{-2 k} \nabla \widetilde{\psi_{k} K}(\xi)=\int 2^{-2 k} \nabla \widetilde{\psi_{k}}(\xi-r \omega) \frac{1}{r-1} r^{n-1} d r d \omega \\
& =\int f_{k}\left((1+d) e_{1}-r \omega\right) \frac{(1+d) e_{1}-r \omega}{r-1} r^{n-1} d r d \omega
\end{aligned}
$$

uniformly in $d$. We write $(1+d) e_{1}-r \omega=(1-r) \omega+d e_{1}+\omega r$. Hence, it is controlled by

$$
\begin{aligned}
O(1) & +\int\left(d e_{1}+\omega\right) \int_{|r-1|<\frac{|d|+\left|\omega-e_{1}\right|}{4}} \frac{f_{k}\left((1+d) e_{1}-r \omega\right)}{r-1} d r d \omega \\
& +\int\left(d e_{1}+\omega\right) \int_{|r-1|>\frac{|d|+\left|\omega-e_{1}\right|}{4}} \frac{f_{k}\left((1+d) e_{1}-r \omega\right)}{r-1} d r d \omega .
\end{aligned}
$$


The third term is uniformly bounded and the second one is bounded by

$$
\begin{array}{r}
\int\left(|d|+\left|\omega-e_{1}\right|\right) \int_{|r-1|<\frac{|d|+\left|\omega-e_{1}\right|}{4}} \frac{\left|f_{k}\left((1+d) e_{1}-r \omega\right)-f_{k}\left((1+d) e_{1}-\omega\right)\right|}{|r-1|} d r d \omega \\
\leq C \int\left(d+\left|\omega-e_{1}\right|\right)^{2} \frac{2^{k} 2^{k n}}{\left[1+2^{k}\left(|d|+\left|\omega-e_{1}\right|\right)\right]^{N}} d \omega \leq C .
\end{array}
$$

Hence, it is enough to prove that $\int \psi_{k}\left(e_{1}-y\right) \frac{1}{|y|-1} d y=O(1)$. The estimate is trivial in the region $|r-1|>1 / 20$ and in the region $\left|\omega-e_{1}\right|>1 / 20$, hence, we only concentrate on

$$
\int_{\substack{|r-1|<1 / 20 \\\left|\omega-e_{1}\right|<1 / 20}} \widetilde{\psi}_{k}\left(e_{1}-r \omega\right) \frac{1}{r-1} r^{n-1} d r d \omega
$$

The term $r^{n-1}$ can be replaced by 1 modulo bounded error terms. Also, we can parametrize $\omega$ by $\omega=\left(\left(1-|\alpha|^{2}\right)^{1 / 2}, \alpha\right)$ where $\alpha \in \mathbb{R}^{n-1}$. Hence, modulo bounded error terms, (A.11) can be written

$$
\int_{\substack{|r-1|<1 / 20 \\|\alpha|<1 / 20}} \widetilde{\psi}_{k}\left(1-r\left(1-|\alpha|^{2}\right)^{1 / 2},-r \alpha\right) \frac{1}{r-1} d r J(\alpha) d \alpha,
$$

where $J(\alpha)=1+O\left(|\alpha|^{2}\right)$ is the Jacobian of the transformation $\alpha \rightarrow \omega$. Next, we write

$$
\begin{aligned}
\widetilde{\psi}_{k}(1- & \left.r\left(1-|\alpha|^{2}\right)^{1 / 2},-r \alpha\right)=\psi_{k}(1-r,-\alpha) \\
& +\int_{1}^{r} \alpha \cdot \frac{\partial \widetilde{\psi}_{k}}{\partial \alpha}(1-r,-t \alpha) d t+\int_{1}^{\left(1-|\alpha|^{2}\right)^{1 / 2}} \frac{\partial \widetilde{\psi}_{k}}{\partial e_{1}}(1-s r,-r \alpha) d s(\mathrm{~A}
\end{aligned}
$$

Hence, (A.12) can be written as the sum of three terms. The first term vanishes

$$
\int_{\substack{|r-1|<1 / 20 \\|\alpha|<1 / 20}} \widetilde{\psi}_{k}(1-r,-\alpha) \frac{J(\alpha)}{r-1} d r d \alpha=0
$$

because $\widetilde{\psi}_{k}$ is radially symmetric and hence the integrand is odd in $r-1$.

The second term is controlled by

$$
\int_{\substack{|r-1|<1 / 20 \\|\alpha|<1 / 20}} \sup _{t \in[1, r]}\left|\nabla \widetilde{\psi}_{k}(1-r,-t \alpha)\right||\alpha| d r d \alpha \leq \sum_{j \geq 0} \frac{2^{k} 2^{n k} 2^{-k+j}\left(2^{-k+j}\right)^{n}}{\left[1+2^{k}\left(2^{-k+j}\right)\right]^{N}} \leq C
$$

where $t \in[1, r]$ should be replaced by $t \in[r, 1]$ if $r<1$ and we split the integral into regions where $|r-1|+|\alpha| \sim 2^{-k+j}$ with $j \geq 0$ (when $j=0$, the region is $\left.|r-1|+|\alpha| \leq 2^{-k}\right)$.

The third term is given by

$$
\int_{\substack{|r-1|<1 / 20 \\|\alpha|<1 / 20}} \int_{1}^{\left(1-|\alpha|^{2}\right)^{1 / 2}} \frac{\partial \widetilde{\psi}_{k}}{\partial e_{1}}(1-s r,-r \alpha) \frac{1}{r-1} d s d r d \alpha
$$


As above, we write $\frac{\partial \widetilde{\psi}_{k}}{\partial e_{1}}(1-s r,-r \alpha)=2^{2 k} f_{k}(1-s r,-r \alpha)(1-s r)$ where $\left|f_{k}(\eta)\right| \lesssim$ $\frac{2^{k n}}{\left(1+2^{k}|\eta|\right)^{N}}$ and $\left|f_{k}^{\prime}(\eta)\right| \lesssim \frac{2^{k} 2^{k n}}{\left(1+2^{k}|\eta|\right)^{N}}$. Hence, using that $1-s r=s(1-r)+1-s$, it is enough to control

$$
\int_{\substack{|r-1|<1 / 20 \\|\alpha|<1 / 20}} \int_{1}^{\left(1-|\alpha|^{2}\right)^{1 / 2}} 2^{2 k}\left|f_{k}(1-s r,-r \alpha)\right| d s d r d \alpha
$$

and

$$
\int_{\substack{|r-1|<1 / 20 \\|\alpha|<1 / 20}} \int_{1}^{\left(1-|\alpha|^{2}\right)^{1 / 2}} 2^{2 k} f_{k}(1-s r,-r \alpha) \frac{1-s}{r-1} d s d r d \alpha
$$

For (A.19), we split the integral in $r$ into two parts: The part where $|r-1|<\frac{|1-s|}{4}$ and the part where $|r-1|>\frac{|1-s|}{4}$. The integral on the second part is dominated by (A.18). For the first part, we use that the integrand can be replaced with

$$
2^{2 k}(1-s) \frac{f_{k}(1-s r,-r \alpha)-f_{k}(1-s,-\alpha)}{r-1},
$$

which is bounded by

$$
2^{2 k}|1-s| \frac{2^{k} 2^{n k}}{\left[1+2^{k}(|1-s|+|\alpha|)\right]^{N}},
$$

where we used that $|1-s r| \geq \frac{1}{3}|1-s|$. Integrating in $r$ and then in $s$, we get a term which is controlled by

$$
\int_{|\alpha|<1 / 20} \frac{2^{2 k} 2^{k} 2^{k n}|\alpha|^{4}}{\left[1+2^{k}|\alpha|\right]^{N}} d \alpha \leq C
$$

It remains to estimate (A.18). We write the integral as sum where $\alpha$ and $r$ are in regions where $|\alpha|+\left|\frac{1}{s}-r\right| \sim 2^{-k+j}$. Hence, we get

$$
\sum_{j} \int_{\left|r-\frac{1}{s}\right|+|\alpha| \sim 2^{-k+j}} 1_{\left(1-|\alpha|^{2}\right)^{1 / 2}<s<1} 2^{2 k} f_{k}(1-s r,-r \alpha) \frac{1-s}{r-1} d s d r d \alpha .
$$

which is controlled by

$$
\sum_{j \geq 0} \frac{2^{2 k} 2^{n k}\left(2^{-k+j}\right)^{2}\left(2^{-k+j}\right)^{n}}{\left[1+2^{k}\left(2^{-k+j}\right)\right]^{N}} \leq C
$$

This ends the proof of the claim (A.4), and so the lemma as well as the remark after it. 


\section{A.1 Alternative proof}

It is a natural question if we can get the same estimate by using the real interpolation. In fact we can do that by the bilinear real interpolation, which gives a subtly better estimate for

$$
\mathcal{A}_{\gamma}^{+}:=\frac{|\nabla|}{|\nabla|-\gamma+i 0} .
$$

Since $\mathcal{A}_{\gamma}-\mathcal{A}_{\gamma}^{+} / 2$ is a regular Fourier multiplier, we get the same bound for $\mathcal{A}_{\gamma}$.

Lemma A.3. Let $n \geq 2$ and $\gamma>0$. If $\operatorname{supp} f \subset\{|\xi| \leq R\}$, then there exists $C(\gamma, R, n)>0$ such that

$$
\left\|\mathcal{A}_{\gamma}^{+} f\right\|_{L^{2(n+1) /(n-1), 2\left(\mathbb{R}^{n}\right)}} \leq C(\gamma, R, n)\|f\|_{L^{2(n+1) /(n+3), 2\left(\mathbb{R}^{n}\right)}} .
$$

Proof. We may assume that $\operatorname{supp} f \subset\{\gamma / 2<|\xi|<\gamma\}$ since outside of it the operator is a regular Fourier multiplier. Here we use a decomposition with respect to time frequency:

$$
\begin{aligned}
-i \mathcal{A}_{\gamma}^{+} f & =\lim _{T \rightarrow \infty} \int_{0}^{T}|\nabla| e^{i(|\nabla|-\gamma) t} f d t \\
& =\int_{0}^{1}|\nabla| e^{i(|\nabla|-\gamma) t} f d t+\sum_{k \in 2^{\mathbb{N}}} \int_{k / 2}^{k}|\nabla| e^{i(|\nabla|-\gamma) t} f d t \\
& =: A_{0} f+\sum_{k \in 2^{\mathbb{N}}} A_{k} f .
\end{aligned}
$$

The first term is bounded by the wave decay estimate:

$$
\left\|A_{0} f\right\|_{L^{2(n+1) /(n-1), 2}} \lesssim \int_{0}^{1} t^{-(n-1) /(n+1)}\|f\|_{L^{2(n+1) /(n+3), 2}} d t \lesssim\|f\|_{L^{2(n+1) /(n+3), 2} .}
$$

To sum the rest, we prove the following estimates:

$$
\begin{aligned}
\left\|A_{k} f\right\|_{L^{\infty}} \lesssim k^{(1-n) / 2}\|f\|_{L^{1}}, \\
\left\|A_{k} f\right\|_{L^{2(n+1) /(n-1)}} \lesssim k^{1 / 2}\|f\|_{L^{2}}, \\
\left\|A_{k}^{*} f\right\|_{L^{2(n+1) /(n-1)}} \lesssim k^{1 / 2}\|f\|_{L^{2}} .
\end{aligned}
$$

For the $L^{\infty}$ estimate, let $\varphi \in \mathcal{S}$ be radially symmetric, supp $\widetilde{\varphi}$ compact away from 0 , and $\widetilde{\varphi}(\xi)=1$ on supp $\tilde{f}$. The support property implies that

$$
\left\|A_{k} f\right\|_{L^{\infty}}=\left\|f * A_{k} \varphi\right\|_{L^{\infty}} \leq\|f\|_{L^{1}}\left\|A_{k} \varphi\right\|_{L^{\infty}}
$$

and by the Fourier transform we have

$$
A_{k} \varphi(r)=C_{n} \int_{k / 2}^{k} \int_{-1}^{1} \int_{0}^{\infty} e^{i(\rho-\gamma) s} \widetilde{\varphi}(\rho) e^{i \rho r c}\left(1-c^{2}\right)^{(n-3) / 2} \rho^{n} d \rho d c d s .
$$


For $r<k / 3$, we can integrate by parts on the phase $e^{i \rho(s+r c)}$ in $\rho$ for arbitrary $N$ times, which gives us a factor $|(s+r c)|^{-N} \lesssim k^{-N}$, and hence

$$
\left|A_{k} \varphi(r)\right| \lesssim k^{1-N}, \quad(r<k / 3)
$$

For $r>k / 3$, we decompose the integral into $|s+r c| \ll 1$ and the rest. Integrating by parts in $\rho$ twice for $|s+r c| \gtrsim 1$, we get a factor $\langle s+r c\rangle^{-2}$, which gives integrability for $s$. Then the integral in $c$ on ||$c|-1| \leq \varepsilon / r$ is bounded by $(1 / r)^{(n-3) / 2+1} \sim$ $r^{-(n-1) / 2} \lesssim k^{(1-n) / 2}$. For the integral on ||$c|-1| \geq \varepsilon / r$, we integrate by parts on the phase $e^{i \rho r c}$ in $c$ for $j$ times with $j \geq(n-1) / 2$, which gives the same factor $(1 / r)^{-1+(n-3) / 2} \lesssim k^{(1-n) / 2}$.

The above argument for $r>k / 3$ works without using the restriction $s \sim k$, hence we get also

$$
\left|\mathcal{A}_{\gamma}^{+} \varphi(x)\right| \lesssim|x|^{(1-n) / 2}
$$

For the $L^{2}$ bound, we use the Tomas-Stein restriction estimate

$$
\left\|\mathcal{F}^{-1} \delta(|\xi|-1) \varphi(\xi)\right\|_{L^{2(n+1) /(n-1)}} \leq C_{n}\|\varphi(\xi)\|_{L^{2}(|\xi|=1)}
$$

By rescaling, we get

$$
\left\|\mathcal{F}^{-1} \delta(|\xi|-\rho) \varphi(\xi)\right\|_{L^{2(n+1) /(n-1)}} \leq C_{n} \rho^{n(n+3) /(2(n+1))-1}\|\varphi(\rho \xi)\|_{L^{2}(|\xi|=1)}
$$

where the exponent on $\rho$ is not important because supp $\widetilde{f}$ is compact. Since

$$
A_{k} f=\mathcal{F}^{-1} \int \rho \frac{e^{i(\rho-\gamma) k}-e^{i(\rho-\gamma) k / 2}}{i(\rho-\gamma)} \delta(|\xi|-\rho) \tilde{f}(\xi) d \rho,
$$

applying the above bound to the integrand we get

$$
\left\|A_{k} f\right\|_{L^{2(n+1) /(n-1)}} \lesssim \int_{0}^{R}\left|\frac{e^{i(\rho-\gamma) k / 2}-1}{\rho-\gamma}\right| \rho^{n(n+3) /(2(n+1))}\|\tilde{f}(\rho \xi)\|_{L^{2}(|\xi|=1)} d \rho,
$$

and then by Schwarz

$$
\begin{aligned}
\left\|A_{k} f\right\|_{L^{2(n+1) /(n-1)}}^{2} & \lesssim \int_{0}^{R}\left|\frac{e^{i(\rho-\gamma) k / 2}-1}{\rho-\gamma}\right|^{2} \rho^{(n+3) /(n-1)} d \rho\|\widetilde{f}\|_{L^{2}\left(\mathbb{R}^{n}\right)}^{2} \\
& \lesssim R^{(n+3) /(n-1)} k \int_{\mathbb{R}}\langle\rho-\gamma\rangle^{-2} d \rho\|f\|_{L^{2}}^{2} .
\end{aligned}
$$

Obviously, the above argument applies also to $A_{k}^{*}$, just by switching the sign of the phase. Thus we get the desired $L^{2(n+1) /(n-1)}$ estimates. 
The estimates (A.29) can be regarded as the bilinear estimates

$$
\left\|\left\langle A_{k} f \mid g\right\rangle_{x}\right\|_{\ell_{\infty}^{s}} \lesssim\|f\|_{L^{p}}\|g\|_{L^{q}}, \quad s=\frac{n+1}{2}\left(\frac{1}{p}+\frac{1}{q}-1\right)-1,
$$

for $(p, q)=(1,1),(2(n+1) /(n+3), 2),(2,2(n+1) /(n+3))$, where $\ell_{q}^{s}$ is the weighted $\ell^{q}$ on $2^{\mathbb{N}}$ with the norm $\left\|a_{k}\right\|_{\ell_{q}^{s}}=\left\|k^{s} a_{k}\right\|_{\ell q\left(k \in 2^{\mathbb{N}}\right)}$. By the complex interpolation, it is extended to inside the triangle spanned by those three points in the $(1 / p, 1 / q)$ plane. In particular, if we define $p_{0}, p_{1}$ by $1 / p_{0}=1 / 2+1 /(n+1)+\varepsilon$ and $1 / p_{1}=$ $1 / 2+1 /(n+1)-2 \varepsilon$, we have the above estimate with $(p, q)=\left(p_{0}, p_{0}\right),\left(p_{0}, p_{1}\right),\left(p_{1}, p_{0}\right)$ for small $\varepsilon>0$, with $s=(n+1) \varepsilon,-(n+1) \varepsilon / 2,-(n+1) \varepsilon / 2$ respectively.

Then by the bilinear real interpolation, we get

$$
\left\|\left\langle A_{k} f \mid g\right\rangle_{x}\right\|_{\left(\ell_{\infty}^{(n+1) \varepsilon}, \ell_{\infty}^{-(n+1) \varepsilon / 2}\right)_{2 / 3,1}} \lesssim\|f\|_{\left(L^{p}, L^{q}\right)_{1 / 3,2}}\|g\|_{\left(L^{p}, L^{q}\right)_{1 / 3,2}}
$$

and by the interpolation property of the $L^{p}$ spaces, it is equivalent to

$$
\sum_{k \in 2^{\mathbb{N}}}\left|\left\langle A_{k} f \mid g\right\rangle_{x}\right| \lesssim\|f\|_{L^{2(n+1) /(n+3), 2}}\|g\|_{L^{2(n+1) /(n+3), 2}}
$$

which implies by duality

$$
\left\|\sum_{k \in 2^{\mathbb{N}}} A_{k} f\right\|_{L^{2(n+1) /(n-1), 2}} \lesssim\|f\|_{L^{2(n+1) /(n+3), 2}},
$$

as desired.

\section{References}

[1] H. Added and S. Added, Equations of Langmuir turbulence and nonlinear Schrödinger equation: smoothness and approximation. Funct. Anal. 79 (1988), no. $1,183-210$.

[2] P. Bechouche, N. Mauser and S. Selberg, Nonrelativistic limit of Klein-GordonMaxwell to Schrödinger-Poisson. Amer. J. Math. 126 (2004), no. 1, 31-64.

[3] L. Bergé, B. Bidégaray and T. Colin, A perturbative analysis of the timeenvelope approximation in strong Langmuir turbulence. Phys. D 95 (1996), no. 3-4, 351-379.

[4] J. Bergh and J. Löfström, Interpolation spaces, An introduction. Grundlehren Math. Wiss. 223, Springer, Berlin-Heiderberg-New York, 1976.

[5] T. Cazenave and F. Weissler, The Cauchy problem for the critical nonlinear Schrödinger equation in $H^{s}$. Nonlinear Anal. 14 (1990), no. 10, 807-836. 
[6] T. Colin, G. Ebrard, G. Gallice and B. Texier, Justification of the Zakharov model from Klein-Gordon-wave systems. Comm. Partial Differential Equations 29 (2004), no. 9-10, 1365-1401.

[7] R. O. Dendy, Plasma Dynamics, Oxford University Press, 1990.

[8] J. Ginibre, Y. Tsutsumi and G. Velo, On the Cauchy problem for the Zakharov system. J. Funct. Anal. 151 (1997), no. 2, 384-436.

[9] C. Kenig, G. Ponce and L. Vega, On the Zakharov and Zakharov-Schulman systems. J. Funct. Anal. 127 (1995), no. 1, 204-234.

[10] S. Machihara, K. Nakanishi and T. Ozawa, Nonrelativistic limit in the energy space for nonlinear Klein-Gordon equations. Math. Ann. 322 (2002), no. 3, 603-621.

[11] N. Masmoudi and K. Nakanishi From nonlinear Klein-Gordon equation to a system of coupled nonlinear Schrödinger equations, Math. Ann. 324 (2002), no. 2, 359-389.

[12] N. Masmoudi and K. Nakanishi, Nonrelativistic limit from Maxwell-KleinGordon and Maxwell-Dirac to Poisson-Schrödinger, Int. Math. Res. Not. 2003, no. $13,697-734$.

[13] N. Masmoudi and K. Nakanishi, From the Klein-Gordon Zakharov system to the nonlinear Schrödinger equation, J. Hyperbolic Differ. Equ. 2 (2005), no. 4, 975-1008.

[14] N. Masmoudi and K. Nakanishi, Energy convergence of singular limits of Zakharov type systems, Invent. Math. 172, (2008), no. 3, 535-583.

[15] N. Masmoudi and K. Nakanishi, From the Klein-Gordon Zakharov system to a singluar Schrödinger system, preprint (2008). Available at http://www.math.nyu.edu/faculty/masmoudi/index.html

[16] T. Ozawa, K. Tsutaya, and Y. Tsutsumi, Well-posedness in energy space for the Cauchy problem of the Klein-Gordon-Zakharov equations with different propagation speeds in three space dimensions. Math. Ann. 313 (1999), no. 1, 127-140.

[17] T. Ozawa and Y. Tsutsumi, The nonlinear Schrödinger limit and the initial layer of the Zakharov equations. Differential Integral Equations 5 (1992), no. 4, $721-745$. 
[18] S. Schochet and M. Weinstein, The nonlinear Schrödinger limit of the Zakharov equations governing Langmuir turbulence. Comm. Math. Phys. 106 (1986), no. 4, 569-580.

[19] C. Sulem and P. L. Sulem, The nonlinear Schrödinger equation. Self-focusing and wave collapse. Applied Mathematical Sciences, 139. Springer-Verlag, New York, 1999.

[20] B. Texier, WKB asymptotics for the Euler-Maxwell equations. to appear in Asymptot. Anal.

[21] T. Tao, Restriction theorems and applications, 1999 available at http://www.math.ucla.edu/

[22] V. E. Zakharov, Collapse of Langmuir waves, Sov. Phys. JETP 35, 908-914. 